\title{
Diskursivität / Historizität von Genre
}

Der Begriff ,Genre' bezeichnet in der ursprünglichen Sprachbedeutung vom lateinischen ,genus ' oder französischen ,genre' abstammend Arten, Sorten und Gattungen. ${ }^{1}$ Seine Begriffsgenese lässt sich weit in die Kunst- und Literaturgeschichte zurückverfolgen. In den Philologien und Geisteswissenschaften bildet der Begriff ,Genre' inzwischen ein disziplinübergreifendes Konzept für Systematisierungen und Konzeptualisierungen diverser Textformen und ein wesentliches Prinzip, um Prozesse der Gruppenbildung von Texten zu beschreiben. Trotz der disziplinübergreifenden Nutzung des Genrebegriffs und der theoretischen wie methodischen Vielfalt von Ansätzen zur Beschreibung von Genres setzt eine systematische theoretische Auseinandersetzung mit Filmgenres erst relativ spät, Ende der 1960er Jahre, in der angloamerikanischen Forschung ein (Kuhn et al. 7). Bis sich theoretische Genrekonzepte also etablieren, dauert es, obwohl der Begriff des ,Genre“ „,bereits in den Jahren um 1910 zum kulturindustriellen Prinzip und damit zur filmkulturellen Institution (avancierte)" (Schweinitz, 101). Mit dem Untersuchungskorpus greift auch die vorliegende Studie dieser genretheoretischen Systematisierung historisch voraus, denn als Psycho 1960 in die Kinos kommt, scheint er aus einer theoriehistorischen Perspektive heraus in einen ,diskursiven Leerraum' einzutreten. Über eine filmhistorische Perspektive wird aber deutlich, dass Psycho evidenterweise im Umfeld von Genrekonventionen wahrgenommen wurde und ,praktische' Genrediskurse avant la lettre prägte, wie unter

\footnotetext{
${ }^{1}$ Die Unterscheidung zwischen Genre und Gattung, wie sie Knut Hickethier zieht, wird in der vorliegenden Studie nicht weiter verfolgt. Da sich die vorliegende Arbeit auf den fiktionalen Bereich eingrenzt und sich das Untersuchungskorpus im Kontext des Hollywoodsystems verortet, wird nur der Genrebegriff verwendet. Zum Verhältnis von Genre und Gattung siehe Hickethier („Genretheorie“), Hißnauer (insbesondere 139-168) und Mundhenke.
}

K. Kirsten, Genresignaturen, Neue Perspektiven der Medienästhetik, https://doi.org/10.1007/978-3-658-36161-7_2 
anderem der Produktionskontext und die Diskurse der Filmkritik und Werbekampagne in Kap. 3 zeigen. $^{2}$ Aufgrund dieser Differenz zwischen Theorie und Praxis, die nicht nur Psycho, sondern Genres allgemein kennzeichnet, wird den Prämissen der Diskursivität und Historizität von Genres eine Theoriegeschichte der Genretheorie vorangestellt. Während die diskursive und historische Verfasstheit von Genres für die einzelnen Fallanalysen des Untersuchungskorpus sowie die gemeinsame Genregeschichte von Psycho, den Sequels Psycho II-IV und der Prequel-Serie Bates Motel von zentraler Erkenntnis sind, ermöglicht die Theoriegeschichte eine theoriehistorische Perspektivierung der gesamten Studie und ihres genretheoretischen Beitrags.

In einem ersten Schritt erfolgt also ein Überblick über die Theoriegeschichte der Genretheorie, ihre historische Entwicklung und theoretischen Felder, in die auch Operationsebenen des Untersuchungskorpus verortet werden. Zugleich verdeutlicht der Überblick, dass sich Genres nicht nur mit unterschiedlichen methodischen Ansätzen aus verschiedenen Disziplinen beschreiben lassen, sondern dass von generischen Phänomenen eine Komplexität ausgeht, die nicht mit einem Ansatz zu erfassen ist. Vor diesem Hintergrund werden für die vorliegende Arbeit nicht nur ein Ansatz und eine Methodik ausgewählt, sondern es wird ein Theorie- und Methodendesign entwickelt, dass sich aus der Genretheorie wie aus einem Baukasten bedient, um die Vielschichtigkeit des Untersuchungskorpus adäquat zu erfassen. Das bedeutet einerseits, dass die diskursiven wie historischen Ebenen von Genres verdeutlicht werden, auf denen das Korpus untersucht wird, und anderseits, dass verschiedene theoretische Ansätze und Zugänge herangezogen werden, um diese Ebenen beschreibbar zu machen und für die Analyse zu operationalisieren. Der zweite Schritt besteht darin, Genres als diskursive Größen zu erarbeiten, indem die verschiedenen Diskurse von Genres vorgestellt werden, bevor die für die Diskursivität von Genres zentralen Aspekte beleuchtet werden. Diese umfassen zum einen die distributionsseitig gelagerten Diskurse der Vermarktung und Werbung, die eine Schnittstellenposition zwischen Produktion und Rezeption einnehmen und für Genres eine evidente Sichtbarkeit herstellen, sowie das Verhältnis von Genreproduktion und Genrekonzept, zwischen dem Genrediskurse wechselseitig vermitteln. Zum anderen werden ein generisches Textverständnis entlang von Genremarkierungen und ein kulturelles

\footnotetext{
2 Ähnliches gilt für die Sequels, wenn auch nicht in diesem offensichtlichen Ausmaß. Neben den systematischen Genretheorien existiert seit den 1970ern ein genretheoretischer Diskurse zum Horrorfilm (u. a. Evans; Kaminsky 130-154; Wood, „Return of the“ und „A Introduction to"). Allerdings konzentriert sich dieser vor allem auf Klassiker wie Psycho. Noch 1987 werden bespielsweise Slasherfilme als „,degenerate aberration“ kaum beachtet (Clover, „Her Body“ 187).
} 
Genreverständnis entlang eines prototypenbasierten Genrebewusstseins entwickelt, bevor zuletzt die darin zentralen Operationsverfahren der Wiederholung und Variation behandelt werden. Im dritten Schritt werden Genres als historische Phänomene beleuchtet, die aus einem distributionsseitigen Bezeichnungsbedürfnis heraus medienübergreifend entstanden sind. Neben der Bezeichnungsebene von Genres wird hierbei die Anwendbarkeit von historischen Modellen, die Genreentwicklungen aus einer Makroperspektive beschreiben, und ,exklusiven` Ansätzen diskutiert, die von Iterationsmustern ausgehen. Dabei wird verdeutlicht, inwiefern für das Untersuchungskorpus von Psycho, den Sequels Psycho II-IV und Bates Motel ein solch ,exklusiver' Ansatz geeignet ist. Schließlich werden in einem abschließenden Schritt die ausgemachten Diskurse und Ebenen und herangezogenen Ansätze vor dem Hintergrund eines ,Baukastens ‘ der Genretheorie diskutiert, die in dem Konzept der Genresignaturen zu einem analytischen Fokus zusammengeführt werden.

\subsection{Theoriegeschichte}

Bevor detaillierter auf die historische Entwicklung von Genrekonzeptualisierungen eingegangen wird, wird ein kurzer Überblick über die Felder der Genretheorie gegeben.

\subsubsection{Felder der Genretheorie}

Während sich diese ersten Genrestudien in den 1940er Jahren vornehmlich auf Einzelgenres konzentrieren und strukturalistisch operieren, um Genrestrukturen als innere Wesenseigenschaften von Filmen festzumachen, entwerfen die darauffolgenden ,systematisch“ angelegten Genrestudien erste Klassifikationen und Ordnungsmodelle, mittels derer sich die Perspektive bereits auf übergreifende Zusammenhänge öffnet. Über strukturalistische und ideologiekritische Zugänge werden so in den 1960er Jahren Genres als Textklassen konzipiert, bevor ab den 1970er Jahren poststrukturalistische Theorien eine pragmatische und intertextuelle Dimension von Genres betonen und psychoanalytisch-feministische Theorien kritisch die Positionierung weiblicher Figuren im Genrekino diskutieren. Im Zuge des durch die New Film History auch in der Genretheorie bewirkten historical turn gewinnt ab den 1980er Jahren ein prozessual angelegter Genrebegriff an Konjunktur, mit dem durch kognitivistische und phänomenologische Ansätze in den 1990er Jahren verstärkt die Filmrezeption und Wahrnehmungsdimension in 
den Fokus rücken. Erst diese breite Hinwendung zu kulturellen Funktions- und Gebrauchsweisen von Genres bringt die diachrone Dynamik, strukturelle Variabilität und systematische Steuerung von Genres zum Vorschein. Daraus lassen sich zwei Beobachtungen ableiten: Einerseits verschiebt sich der Blickwinkel von textimmanenten Studien, die Eindeutigkeiten, Klassifikationen und Evolutionen suchen, auf Diskursszenarien und Wandlungsprozesse, womit sich nicht nur ein anti-essentialistisches Genreverständnis durchsetzt, dem sich auch die vorliegende Arbeit anschließt, sondern es geraten diverse Anwendungsfelder und Funktionszusammenhänge von Genres in den Fokus. Diese können in der folgende Theoriegeschichte zwar nicht vollständig abgebildet werden, aber entlang zentraler fachhistorischer Linien nachvollzogen werden. Anderseits lassen sich hier schon drei grobe Felder der Genretheorie differenzieren, die auf jeweils unterschiedlichen Abstraktionsniveaus argumentieren. ${ }^{3}$ Neben der Gruppierung von ähnlichen Filmen zu einer ,Genrefamilie', wie es erste Genrestudien als Einzeltheorien von Genres (1) in den 1940er Jahren vornehmen, um familiäre Beziehungen, gemeinsame Merkmale und historische Entwicklungen zu untersuchen, beschäftigen sich allgemeine Genretheorien (2) ab den 1960er Jahren mit dem Begriff, dem Konzept und der Anwendbarkeit von Genres auf einer den Einzeltheorien übergeordneten Metaebene. Hierbei fokussieren genresystematische Herangehensweisen vor allem die Verhältnisse zwischen einzelnen Genres in kulturellen und historischen Ausprägungen, während sich metaperspektivisch angelegte Ansätze dem Genre als mediale Ordnungsform und kulturelles Prinzip zuwenden. Dabei orientieren sich allgemeine Genretheorien stets auch an Einzeltheorien, indem sie Erkenntnisse zu einzelnen Genres in eine abstrakte Genretheorie überführen oder exemplarisch an einzelnen Genres ansetzen. Umgekehrt greifen ebenso Einzeltheorien Modelle und Prinzipien aus allgemeinen Genretheorien auf. Zudem sind bei Einzeltheorien zwei wesentliche Prinzipien der Korpusbildung zu unterscheiden. Ausgehend von einer weit gefassten, genrespezifischen Minimaldefinition integriert ein inklusiver Korpus all jene Produktionen, die dieser Definition entsprechen, sodass auch periphere Bereiche des Genres einfließen. Dagegen stellt eine exklusive Gruppe einen ,Kern' des Genres auf, der Produktionen entlang einer engen Definition aufnimmt und häufig kanonisierte Produktionen oder auch Prototypen umfasst (Kuhn et al. 19). ${ }^{4}$

\footnotetext{
${ }^{3}$ Eine ausführliche Übersicht zu den Dimensionen der Genretheorie findet sich in Kuhn et al., 24-29.

${ }^{4}$ Zur Zirkularität der Korpusbildung siehe Abschn. 2.2.2. Zur Funktion von Prototypen siehe Abschn. 2.2.3.
} 
Schließlich lassen sich noch genretheoretische Überlegungen anhand von Einzelanalysen (3) finden, um auf einer Mikroebene Fallbeispiele zu analysieren, die beispielsweise idealtypische Vertreter eines Genres darstellen oder als ,Blaupause" einzelne Genres erweitert oder neu ausgerichtet haben. Inwiefern sich die Analysen des Untersuchungskorpus in diese Felder einordnen lassen und welche Ansätze dafür herangezogen werden, wird am Ende des folgenden Abschnitts verdeutlicht.

\subsubsection{Entwicklung von Genrekonzeptualisierungen}

Die Schriften der frühen Filmtheorie aus den 1920er und 1930er Jahren in Deutschland zeichnen sich vor allem durch eine ablehnende Haltung gegenüber Genreproduktionen aus, denen sie als populäre Massenware ohne ,originären Kern' eine Formelhaftigkeit und Stereotypie vorwerfen. Insgesamt haben Genres als Symbol der Massenunterhaltung in der Weimarer Republik keinen guten Stand, da übergreifend der ,künstlerisch anspruchsvolle“ Film als Maßstab gilt. Rudolf Arnheims Überlegungen zum „Konfektionsfilm“ zeigen dies eindringlich. Als Konfektionsfilm bezeichnet er seriell gefertigte Filme, die ohne künstlerische Ambitionen für die anspruchslose und flache Unterhaltung eines Massenpublikums hergestellt werden. Der „konfektionierte Massenfilm“ (270) lenkt von der Ungerechtigkeit der Wirklichkeit ab, indem er eine Welt des Guten und Schönen präsentiert, in der die Schlechten bestraft werden, womit er als „harmlose Unterhaltungsware" die bestehende Gesellschaftsordnung stabilisiert, statt sie zu hinterfragen. In der hohen Nachfrage nach „Konfektionsfilmen“ sieht Arnheim die Gefahr, einen Geschmack der „Durchschnittsmenschen“ zu bedienen und darin „Spießer[n] gefährlichsten Kalibers“ zu gefallen (165-172).

Dies Dumme und Schlechte im Menschen streichelt der Konfektionsfilm; er sorgt dafür, dass die Unzufriedenheit sich nicht in revolutionäre Tat entlade, sondern in Träumen von einer besseren Welt abklinge. Er serviert das Bekämpfenswerte in Zuckerpastillen. (164)

Auch Siegfried Kracauer bezeichnet die seriellen Produktionen seiner Zeit als „Durchschnittsproduktion“ für den gemeinen Massengeschmack, deren ideologischen Einfluss zu brechen er zur Aufgabe des Filmkritikers macht (,Aufgabe des Filmkritikers“, 63). 
Die Filmproduktion hat sich so stabilisiert wie das Publikum. Ihre Erzeugnisse weisen typische, immer wiederkehrende Motive und Tendenzen auf, und selbst die vom Durchschnitt abweichenden Filme bieten kaum noch eine Überraschung. Eine Verfestigung, die sich sowohl auf die Filmfabel wie auf das technische Verfahren erstreckt. [...] Nicht die Typisierung des Films ist verwerflich. [...] Verwerflich ist die Gesinnung der Filme. [...] (U)nsere gesellschaftliche Wirklichkeit (wird) auf bald idiotisch-harmlose, bald verruchte Weise verflüchtigt, beschönigt, entstellt. (,Der heutige Film“, 101)

Diese Überlegungen setzen später Max Horkheimer und Theodor W. Adorno fort und spitzen sie im Rahmen ihrer Diskussion der ,Kulturindustrie“ noch einmal systemkritisch zu, wenn sie vom „Stein der Stereotypie“ sprechen, der „(b)ei allem Fortschritt der Darstellungstechnik, der Regeln und Spezialitäten, bei allem zappelnden Betrieb das Brot (bleibt), mit dem Kulturindustrie die Menschen speist“ (175). Sowohl Kracauer als auch die Vertreter der Kritischen Theorie werfen den Genreproduktionen eine Wirklichkeitsferne vor und unterstellen Genres eine generelle Verblendung, worin sie dem Publikum allerdings eine Unmündigkeit bescheinigen. Die dennoch unternommenen, wenn auch wenigen Versuche, Genreproduktionen an kunsttheoretische Debatten anzuknüpfen, wie durch Béla Balázs und Erwin Panofsky mit Analogien zur vorindustriellen Volkskunst oder zu mittelalterlichen Dombauhütten (Schweinitz, 103), bleiben ohne Wirkung. Bis in die 1960er Jahre hinein dominiert nicht nur in Deutschland die Kunstkino-Perspektive die filmtheoretischen Debatten, die darum bemüht sind, die Kunstfähigkeit des Films zu belegen. Die zahlreichen Genreproduktionen bleiben konträr dazu gesetzt und erhalten kaum eine theoretische Beachtung. Wie Jörg Schweinitz in seiner Skizzierung der Diskursentwicklung verdeutlicht, ist die Kunstdebatte aber der Filmindustrie in ihrer Frühphase durchaus sehr zuträglich gewesen: „Die Kunsttheorie des Films besaß im Lichte solcher Bemühungen der Industrie etwas von einer flankierenden Funktion.“ (Ebd. 102) Denn als sich Mitte der 1910er Jahre der abendfüllende Langspielfilm - der sogenannte feature film - als Produktionsstandard durchsetzt, markiert dies den Höhepunkt der filmindustriellen Bestrebungen, bei möglichst effizienter Standardisierung der Produktion auch eine innovative Neuheit zu schaffen. So wird zwar stets der Charakter des originären Kunstwerks betont, aber zugleich werden Elemente des Bekannten und Bewährten mitbeworben. Dies erfolgt dann häufig durch den Bezug auf bestehende Filmgenres, wie Staiger im frühen Hollywoodkino aufzeigt (,Mass-Produced Photoplays“).

Erste eingehendere Genrediskussionen entstehen in den späten 1940er und frühen 1950er Jahren, als sich vermehrt mit einzelnen Genres auseinandergesetzt wird. Wegweisende Schriften veröffentlichen Robert Warshow in den USA und 
André Bazin sowie Jean-Louis Rieupeyrout in Frankreich. Ihre frühen Genrestudien konzentrieren sich dabei häufig auf den Western und den Gangsterfilm, die während der Hochphase des klassischen Hollywoods überaus populäre Genres darstellen, wie Barry Keith Grant bemerkt: „It is not surprising that Warshow and Bazin focused on westerns and gangster films since these have been perhaps the two most durable of American film genres [...].“ (XVIIf.) In seiner strukturellen Auslegung des Gangsterfilms, nach der das gleiche Muster mit jedem neuen Film wiederholt wird, nimmt Warshow die später folgenden strukturalistischen Ansätze vorweg.

In its initial character, the gangster film is simply one example of the movies' constant tendency to create fixed dramatic patterns that can be repeated indefinitely with reasonable expectation of profit. One gangster film follows another as one musical or one Western follows another. (,Westerner“"12)

Die aus den Kunstdebatten resultierende oppositionelle Paarung von , Kunst vs. Genre", die den Weg für generische Betrachtungen nicht nur in Deutschland für lange Zeit blockiert, verliert bei Warshow nicht nur an Bedeutung, er überführt das dichotome Verhältnis in ein relationales, in dem künstlerische Formen ebenso konventionelle Iterationsstrukturen hervorbringen: „But this rigidity is not necessarily opposed to the requirements of art. There have been very successful types of art in the past which developed such specific and detailed conventions as almost to make individual examples of the type interchangeable." (Ebd. 12) In seinen Überlegungen zum Western geht Bazin noch einen Schritt weiter, indem er die strukturellen Merkmale einer mythologischen Deutung unterzieht und kulturell aufwertet: ,Diese formalen Attribute, an denen man den Western gewöhnlich erkennt, sind nur Zeichen und Symbole dessen, was er in Wirklichkeit ist: Mythos.“ (,Western“ 257) Diese ersten Genrestudien sind von einer valorisierenden Reflexion über einzelne Genres gekennzeichnet, die inspiriert von Roland Barthes' Mythentheorie nicht nur künstlerischen Ursprüngen nachspürt, sondern vor allem Genres als eine ästhetische Verkleidung gesellschaftlicher Mythen begreift („Mythen des Alltags“). Mit ihrem Blick auf die tieferen Schichten populärer Genreunterhaltung legen sie den Grundstein für spätere systematischere Studien, die ab den 1970er Jahren vornehmlich in den USA entstehen und sowohl allgemeine Theoriemodelle als auch exemplarische Einzelstudien hervorbringen, unter anderem zum Musical (Altman, „American Film Musical“), zum Western (Buscombe) und zum Melodram (Neale, „Melo Talks“ und Elsaesser, „Tales of Sound"). 
Voraussetzung dafür, dass sich das Genrekonzept schließlich in der Filmtheorie durchsetzen kann, ist die Abwendung von autor- und werkbezogenen Betrachtungen. Über den Umweg der Auteur-Theorie, die in den 1960er Jahren maßgeblich durch Andrew Sarris in die USA überführt und weiterentwickelt sowie popularisiert wird, wird in Anlehnung an die Cahiers du Cinéma nach den künstlerischen Handschriften der Regisseure innerhalb des amerikanischen Genreproduktionssystems gefragt. Dies ebnet den Weg hin zu einer offeneren Betrachtung der Genreproduktionen Hollywoods („Auteur Theory“). Die dieser Auseinandersetzung noch innewohnende Widersprüchlichkeit, ,einen ,Auteur' selbst dann wie einen klassischen Künstler zu behandeln, wenn er ein Filmgeschehen einrichtet, das aus der Perspektive eines überlieferten Kunstverständnisses eigentlich nicht anders als ,trivial" erscheinen konnte," (Schweinitz, 104) wird erst mit der Entstehung der Filmsemiotik sowie dem kritischen Zeitgeist der späten 1960er Jahre aufgelöst. Bisherige Denkrichtungen und Kunstverständnisse werden infrage gestellt, das Interesse sowohl am Film als Sprachsystem als auch an seinen ideologischen bzw. mythologischen Bedeutungsschichten gewinnt an Konjunktur und befeuert die genretheoretischen Diskussionen, die in den 1970er Jahren erste systematische Beiträge vor allem im angloamerikanischen Forschungskontext hervorbringen. Die strukturalistischen und textimmanent operierenden Ansätze erfassen die Komplexität generischer Merkmale und Strukturen mittels fixierter Systeme (siehe u. a. Cawelti, Buscombe, Kaminsky und Schrader). So bilden Filmgenres beispielweise für Cawelti komplexe Textklassen, die über ein vielgliedriges Invarianzmuster das immer gleiche Strukturmuster variieren und als „structural pattern which embodies a universal life pattern or myth in the materials of language" (387) zu bestimmen sind. Auch diese Diskussionen begreifen die populären Formen des Genrekinos als eine moderne Art der Mythologie, die auf gesellschaftliche Dispositionen antwortet und über genrespezifische Themen, Figuren und Ästhetiken gesellschaftliche Wert- und Normvorstellungen reflektiert.

Neben dieser angloamerikanischen Diskussion verfolgen in den 1970er und 1980er Jahren poststrukturalistische Genretheorien aus Frankreich vor allem pragmatische Definitionsansätze, wie sie unter anderem bei Gérard Genette, Jacques Derrida und Roger Odin zu finden sind. Ähnlich Tzvetan Todorovs Einführung zur fantastischen Literatur entstammen viele dieser Arbeiten der literaturwissenschaftlichen Diskussion, die Genres als intertextuelle Beziehungsgeflechte beschreiben. Todorovs Arbeit zum Fantastischen bildet eine der ersten theoretischen Bestimmungen des Genres, auf die vor allem auch die deutschsprachige 
Diskussion zum Fantastischen aufbaut (u. a. Friedrich, Giesen). Mit Todorov lassen sich Genres im audiovisuellen Kontext als Relais beschreiben, mit denen Filme in historische Kontexte wie die Filmgeschichte eintreten.

D'une manière plus générale, ne pas reconnaître l'existence des genres équivaut à prétendre que l'œuvre littéraire n'entretient pas de relations avec les œuvres déjà existantes. Les genres sont précisément ces relais par lesquels l'œuvre se met en rapport avec l'univers de la littérature. (12)

Viele der französischen Ansätze verorten Genres innerhalb der Lektüre als einen Akt der Identifizierung: Für Genette besitzen Genres eine , architextuelle` Qualität, über die der Text in Beziehung zu anderen Texten tritt (,Palimpseste“ 9). Doch hierbei ist es nicht der Text selbst, der sein Genre bestimmt, sondern die Aufgabe ,des Lesers, des Kritikers, des Publikums“ (ebd. 14), das Genre zuzuordnen. Für diese Personenkreise konzentriert sich in Genres wichtiges transtextuelles Wissen, welches den Erwartungshorizont und die Rezeption von Texten lenkt und bestimmt (ebd.). Auch bei Jean-Marie Schaeffer werden Genres trotz ihrer textuellen Ähnlichkeiten als Klassifikationsgröße der Lektüre konzipiert (,Du texte au genre“ 199 f.). ${ }^{5}$ Dagegen geht Jacques Derrida von Markierungen aus, über die sich Texte auf vielfältige Weise generisch ausweisen und an kulturellen Genrekonventionen teilnehmen, ohne dass diese Markierungen selbst eine genrehafte Spezifik annehmen (,The Law“ 64 f.). Da dieses Verständnis von Genremarkierungen Genres auf der Textebene verortbar macht, ohne von einer Immanenz auszugehen, und sie in Bezug zu kulturellen Konventionen von Genres setzt, wird Derridas Ansatz innerhalb des Theorie- und Methodendesigns der vorliegenden Arbeit zur Beschreibung der textuellen Ebene von Genreproduktionen herangezogen.

Die deutschsprachige Filmwissenschaft entdeckt das Genrekonzept erst Ende der 1970er Jahre. 1979 bringen Bernhard Roloff und Georg Seeßlen mit dem Buch Western-Kino, von Seeßlen und Claudius Weil geschrieben, den ersten Band ihrer Grundlagen des populären Films im Rowohlt-Verlag heraus, in dem sie die bis dato abseits des dominierenden Autorenkinos und innerhalb von Filmclubs geführten Debatten um das Genrekino reflektieren. Bereits zuvor haben sie im Münchner Eigenprogramm Roloff und Seeßlen die ,Typologie, Geschichte und Mythologie“" einiger ,großer" Genres wie der Komödie, des Gangsterfilms

\footnotetext{
${ }^{5}$ Siehe auch den semio-pragmatischen Ansatz von Odin, der die Textproduktion als einen doppelten Prozess erfasst, bei dem ,,der eine sich im Raum der Herstellung (abspielt), der andere in dem der Lektüre“ („Kunst und Ästhetik“ 42), und der die ,Genre-Produktion“ beim Dokumentarfilm als „,dokumentarisierende Lektüre“ beschreibt („Dokumentarischer Film“).
} 
und des erotischen Films aufgearbeitet. Weitere Rowohlt-Bände zur Reihe, die inzwischen in Neuauflagen und Überarbeitungen im Schüren Verlag erscheinen, widmen sich dem Thriller, dem Melodram, dem Horror-, Science-Fiction-, Detektiv-, Abenteuer- und Polizeifilm. Angelehnt an den mythischen Genrebegriff liefern die mehrbändigen Grundlagen des populären Films erste umfangreiche historische Übersichten, die in der deutschen Film- und Fernsehwissenschaft sowohl den Genrebegriff etablieren als auch den Blick nachhaltig auf die Wechselwirkungen zwischen populären Kinoformen und gesellschaftlichen Wertesystemen richten.

Im Gegensatz zu den strukturalistischen Ansätzen setzt sich auch in den angloamerikanischen Genrediskussionen in den 1980er Jahren allmählich die Einsicht durch, dass Genres nicht durch statische Taxonomien festzusetzen sind, sondern vielmehr Familienähnlichkeiten aufweisen, die Wandlungen und Verschiebungen unterliegen. Das geht mit der zentralen Einsich einher, dass es sich als Trugschluss erweist, „daß ein Muster wenigstens zu einem gewissen Zeitpunkt das gesamte Genre restlos beherrschen und eine geschlossene narrative Makrostruktur für das Gesamtgenre bereitstellen würde“ (Schweinitz, 108; Herv.i.O.). Statt also nach festen Mustern eine Art ahistorischer Genreessenz zu suchen, werden Genres vielmehr in ihrer historischen und kulturellen Veränderbarkeit als Phänomene erfasst, die einem steten Wandel unterliegen. Ähnlich Wladimir Propps Strukturmodell, das bereits 1928 für russische Märchen verschiedene Motive als flexibel und variabel wiederkehrend identifiziert und keine übergeordnete, festgeschriebene Makrostruktur aufstellt, lösen sich die Genrekonzepte von einem starren Schematismus und einer essentialistischen Vorstellung und nehmen, wie bei Robert Altman, eine Neuausrichtung genretheoretischer und genrehistorischer Diskussionen vor:

As long as Hollywood genres are conceived as Platonic categories, existing outside the flow of time, it will be impossible to reconcile genre theory, which has always accepted as given the timelessness of a characteristic structure, and a genre history, which has concentrated on chronicling the development, deployment, and disappearance of this same structure. (,Semantic/Syntactic“ 29)

Altman schlägt in Abkehr zu den semiotisch-strukturalistischen Konzepten einen semantisch-syntaktischen Zugang vor, der dual operiert, indem er die semantische Textanalyse mit einer syntaktischen Betrachtung verbindet: „By simultaneously accepting semantic and syntactic notions of genre we avail ourselves of a possible way to deal critically with different levels of , genericity'." (Ebd. 34) Statt der 
semantischen Gesamterscheinung isoliert Altman einzelne Elemente als semantische Einheiten, die viele generische Bedeutungsträger - wie beispielsweise typische Figuren, spezifische Settings und Kameraeinstellungen - umfassen können. Diese werden auf der syntaktischen Ebene eines Films strukturell jeweils neu miteinander kombiniert und organisiert. Daraus leitet Altman das jeweils neue generische Gesamtbild eines Films ab. Später fügt Altman seinem Zugang noch eine pragmatische Dimension hinzu, in der er die diskursive Beschaffenheit von Genres einbindet (,Film/Genre“ 208). In Anerkennung der unterschiedlichen Nutzungsweisen von Genreterminologien, die mitunter in Konkurrenz zueinander treten können, sind Genres ebenso wenig auf einer pragmatischen Ebene festzuschreiben. Vielmehr sind sie über verschiedene Diskurse, Gebrauchsformen und Lesarten zu beschreiben, was auch den Ausgangspunkt des Theorie- und Methodendesigns bildet. Dieser Variabilität und Vielfalt auf semantischer, syntaktischer und pragmatischer Ebene verdanken nach Altman viele Genres ihre Existenz:

Instead of a word or a category capable of clear and stable definitions (the goal of previous genre theorists), genre has here been presented as a multivalent term multiply and variously valorized by diverse user groups. Successful genres of course carry with them an air of user agreement about the nature both of genres in general and of this genre in particular, thus implying that genres are the unproblematic product of user sharing. In fact, the moments of clear and stable sharing typically adduced as generic models represent special cases within a broader general situation of user competition. While genres may make meaning by regulating and coordinating disparate users, they always do so in an arena where users with divergent interest compete to carry out their own programs. (Ebd. 214 f.)

Wegbereitend für weitere Genrestudien, die in den 1980er und 1990er Jahren insgesamt einen neuen Boom an Genretheorien verzeichnen und unter anderem im Film Genre Reader I-IV von Grant nachverfolgt werden können, ist Steve Neales Vorschlag eines prozessualen und kontextuellen Genreverständnisses, den er bereits 1980 (,Genre“) entwirft und in den folgenden Jahrzehnten weiterentwickelt (siehe „Questions of Genre“ und „Genre and Hollywood“). Genres bilden für Neale zentrale narrative Modi innerhalb des filmischen Systems (,Genre“ 20), das er als ,systems of orientations, expectations and conventions that circulate between industry, text and subject" und ,a constantly fluctuating series of signifying processes“ (ebd. 19) beschreibt. Für dieses System bilden Genres prozesshafte Größen: 
Genres, then, are not systems: they are processes of systematization. It is only as such that they can perform the role allotted them by the cinematic institution. It is only as such that they can function to provide, simultaneously, both regulation and variety. (Ebd. 51)

Die prozesshafte Natur von Genres führt er später auf die Interaktion zwischen „the level of expectations, the level of generic corpus, and the level of the ,rules" or ,norms“ that govern both“ (,Questions of Genre“ 189) zurück, womit er die Textebene mit der Produktions- und Rezeptionsseite zusammenführt und Altmans multidimensionale Betrachtung vorwegnimmt. Neale und Altman stehen repräsentativ für einen historical turn innerhalb der Genreforschung, mit dem sich die Prämisse durchgesetzt hat, dass Genres grundlegend einer fortlaufenden Transformation unterliegen. ${ }^{6}$ Da diesem historischen Verständnis auch die vorliegende Arbeit folgt, werden die Ansätze von Altman und Neale innerhalb des Theorieund Methodendesigns für die historische Konzeption von Genres herangezogen.

Parallel zu den prozessualen Genrekonzepten entwickeln sich in den 1990er Jahren vermehrt kognitivistische Ansätze, die von der Psychologie und der allgemeinen Rezeptionstheorie des Films herkommend verstärkt die kognitive, kommunikative und emotionale Steuerung der Filmrezeption durch Genres untersuchen. Innerhalb kognitiver Vorgänge des allgemeinen Filmverstehens werden Genres hinsichtlich ihrer kommunikativen Funktion betrachtet und als Verständigungsinstrumente zwischen Produktion und Rezeption aufgefasst. Daran anschließend werden Genreproduktionen dahingehend befragt, wie sie sich an Zuschauende richten und rezipiert werden (u. a. Casetti; Mikos; Seel/Keppler). ${ }^{7}$ Angesichts der aufscheinenden Konsistenz von Genrezusammenhängen auf Seiten der Rezeption entwickelt Schweinitz in Rückgriff auf einen kognitionspsychologischen Prototypenansatz ein ,filmkulturelles Genrebewusstsein“, das erst „,dem ,Genre-Code“ als Faktor innerhalb des filmkulturellen Diskurses lebendige Existenz (verleiht).“ (113) Schweinitz' Ansatz erfasst die kulturelle Ebene von Genres sowohl über Konventionalisierungen als auch über Prototypen, wie ihn Psycho für den Horrorfilm und Psycho-Thriller bildet. Das Theoriedesign nutzt dieses Konzept, um in Kombination mit Derridas poststrukturalistischem Ansatz eine Textebene von Genres zu erarbeiten, die über Markierungen Konventionen einbindet. Durch die an Konjunktur gewinnenden Kognitionswissenschaften in den 1990er Jahren rücken des Weiteren emotionale Aspekte des Genreerlebens in den Fokus genretheoretischer Betrachtungen, sowohl in der angloamerikanischen

\footnotetext{
${ }^{6}$ Siehe unter anderem Cohen; Hickethier, „Genretheorie“; Jauss und Schweinitz.

${ }^{7}$ Der hier gewählte Sprachgebrauch von ,Zuschauenden“ markiert eine geschlechtsneutrale Analysekategorie jenseits heteronormativer Inskriptionen.
} 
wie etwas später auch in der deutschsprachigen Forschung. Bereits Ende der 1980er Jahre beschäftigt sich Christian Mikunda mit Emotionen im Film, die er aus kognitionstheoretischer Perspektive untersucht. Weitere Arbeiten von Edward Branigan, Peter Wuss, Joseph D. Anderson, Ed S. Tan, Carl Plantinga / Greg M. Smith sowie Hans Jürgen Wulff (,Psychologie und Film“), Anne Bartsch et al. etablieren das kognitive Paradigma innerhalb der Filmwissenschaft und legen damit eine filmtheoretische Grundlage, auf die weitere kognitionstheoretische Arbeiten zu Genres aufbauen (siehe Grodal und Carroll, Emotion). Beispielsweise untersucht Carroll die emotionale Steuerung von Zuschauenden durch Filmgenres, wobei Emotionen nicht mehr als körperliche Effekte neben kognitiven Vorgängen gestellt werden, sondern nunmehr als Teil von Kognitionen verstanden werden. Emotionale Zustände formieren sich erst über das kognitive Erkennen von bestimmten Merkmalen, die den entsprechenden emotionalen Zustand auszeichnen: „In fear, the object must meet the criterion of being harmful or, at least, of being perceived to be harmful.“ (27 ff.). Nach Carroll evozieren Genres eine ganze Bandbreite an Emotionen, wobei bestimmte Genres spezielle emotionale Zustände vorstrukturieren.

That is, whereas all genres tend to evoke anger, joy, hatred, and the like, in addition to these emotions some genres also aim at arousing specific emotions in spectators as a condition of being an instance of the very genre in question. Or, to put it differently, raising various preordained emotions in spectators is the sine qua non of certain film genres. (35)

Phänomenologische Ansätze, deren maßgebliche Theorien zu Beginn der 1990er Jahre erscheinen und richtungsweisende Arbeiten wie Vivianne Sobchacks Phenomenology of Film Experience hervorbringen, führen Genrediskussionen noch weiter und erfassen filmische Effekte insbesondere über körperbezogene Wahrnehmungen, wobei verschiedene körperbezogene Modi adressiert und ein intensives Körpererleben ermöglicht werden. In ihrem psychoanalytischen Theoriemodell der „,body genres“ (,Körper-Genres“) konzipiert Linda Williams eine übergreifende Genretheorie des Horrors, des Melodrams und des pornografischen Films, die entlang generischer und genderspezifischer Konfigurationen des Körpers spezifische generische Affektökonomien entwickeln (,Film Bodies“). Die Spezifik dieser Genres vereint nach Williams eine audiovisuelle Inszenierungsweise, die die materielle Verfasstheit und sinnliche Erscheinung von Körpern in den Vordergrund stellt und über explizite Körperbilder die (Körper-) Wahrnehmung der Zuschauenden affiziert. Dass die Genres auf der Darstellungsebene primär den weiblichen Körper als Figuration des ekstatischen Spektakels von 
Lust, Angst und Schmerz verobjektivieren, setzt Williams sowohl in Bezug zu Freud'schen Urfantasien der Verführung, Kastration sowie des Ursprungs als auch zu spezifischen Perversionen des Sadismus, Sadomasochismus und Masochismus. Beide Seiten finden in den Genres ihre Entsprechung und manifestieren sich insbesondere innerhalb der lüsternen, gequälten und heulenden Frau (ebd. 173). Mit der wirkungsästhetischen Beschäftigung der body genres nimmt Williams schließlich eine Relektüre der häufig als ,niederwertig' angesehenen Genres vor: „these ,gross' body genres, which may seem so violent and inimical to women, cannot be dismissed as evidence of a monolithic and unchanging misogyny, as either pure sadism for male viewers or as masochism for females." (Ebd. 175) Hiermit stellt sie nicht nur die feministische Filmtheorie der 1970er in Bezug auf ihre Kritik an der patriarchalen und misogynen Symbolik in Frage, sondern trägt innerhalb der Genretheorie zu einer erheblichen Komplexitätssteigerung der genre- wie auch gendertheoretischen Diskussionen bei. Indem sie aufzeigt, wie ein genussvolles Filmerleben möglich ist, auch wenn die Zuschauerin mit einer zum Opfer gemachten Frauendarstellung konfrontiert ist, stellt sie einerseits die geschlechtlich uneindeutige Erfahrungsdimension dieser Genres heraus, die zwischen den Wahrnehmungspolen aktiv/passiv, männlich/weiblich und sadistisch/masochistisch vielmehr changieren, als dass sie eindeutige Zuordnungen anbieten. Anderseits wendet sie sich damit auch von bisherigen psychoanalytischen ,Bestrafungsdeutungen ' dieser Genres ab. Da Williams' Theoriemodell der body genres sich sowohl als Einzeltheorie für die Betrachtung der einzelnen Genrekonzepte heranziehen lässt als auch eine allgemeine Theorieperspektive auf phänomenologische Dimensionen von Genres und ihre Affektsteuerung erlaubt, wird dieser Ansatz insbesondere in den Analysen der Sequels herangezogen, um den Schrecken als genrespezifisches Grundmoment zu konturieren, der von den Produktionen immer wieder reaktiviert wird.

Ansätze der feministischen Filmtheorie haben sich bereits in den 1970er Jahren unter dem Einfluss der psychoanalytischen Theorie und Film-Semiotik mit Hollywood, dem Genrekino und der Positionierung von weiblichen Figuren kritisch auseinandergesetzt. Insbesondere das Hollywood-Melodrama bot reiches Material für die feministische Kritik der 1970er Jahre, wie Mulvey rückblickend ausführt: „Anhand dieses Genres, das sich speziell an ein weibliches Publikum richtete, konnten die häusliche Sphäre und Dilemmata, mit denen Frauen als Mütter konfrontiert waren, untersucht werden." (Mulvey 22) Mit den aufkommenden Gender Studies in den 1980er Jahren erhalten diese von Judith Butlers Gender Trouble inspirierten Fragestellungen eine neue Richtung, indem sie Gender als kritische Analysekategorie zu einer zentralen Begrifflichkeit erheben und vermehrt Geschlechterverhältnisse und Darstellungsweisen von Weiblichkeit als 
auch Männlichkeit in Genres untersuchen. Das steigende Interesse der Genretheorie an Gender-Repräsentationen als genrespezifische Konfigurationen spiegelt sich beispielsweise in der Auseinandersetzung mit dem Horrorfilm wider, an dem unter anderem Barbara Creed in Rückgriff auf Kristevas Abjekt-Theorie ihr Konzept des „Monströs-Femininen“ (Monstrous-Feminine) entwickelt. Aber vor allem mit Linda Williams‘ body genres und Carol J. Clovers Untersuchung des Final Girl wurden für die Genre- als auch Gendertheorie wegweisende Arbeiten vorgelegt, die beide Konzepte in produktiver Weise zusammenführen. ${ }^{8}$ Am Beispiel der Slasher-, Rape-Revenge- und Occult-Filme konzipiert Clover eine Gendertheorie des modernen Horrorfilms, in dem das Final Girl nicht nur als einzige die Angriffe des Serienkillers überlebt, sondern die ihr anfänglich zugeschriebene Opferrolle auch noch übersteigt und mit brutaler Heftigkeit zurückschlägt. Sie besiegt ihre Angreifer eigenhändig, während sich die männlichen Figuren als untaugliche Helfer herausstellen. Als eine der wenigen standardisierten weiblichen Heldenfiguren besteht ihre generische Spezifik in der Personifizierung des sowohl zu erleidenden Terrors als auch der attackierenden Gegenwehr, die sich in eine transgressive Gender-Figuration übersetzen und eindeutige Geschlechterzuordnungen negieren:

For if ,masculine' describes the Final Girl some of the time, and in some of her more theatrical moments, it does not do justice to the sense of her character as a whole. She alternates between registers from the outset; before her final struggle she endures the deepest throes of ,feminity '; and even during the final struggle she is now weak and strong, now flees the killer and now charges him, now stabs and is stabbed, now cries out in fear and now shouts in anger. She is a physical female and a characterological androgyne: like her name, not masculine but either/or, both, ambiguous. (,Men, Women“ 63)

Neben Williams wird auch Clovers Theorie für die Analyse der Sequels und von Bates Motel herangezogen, weil sie einerseits von einer breiten Intertextualität ausgeht, die Genres als stete Variationen und schematisch-repetitive Formen ansieht, und anderseits in ihrer Teilstudie zum Slasherfilm Psycho nicht nur als ,,ancestor“ (ebd. 23) den Analysen prominent voranstellt, sondern ausgehend von Psycho's populär gewordenen Genremustern mehrere analytische Bezugspunkte entwickelt. Neben dem Final Girl und den meist sexuell gestörten, infantilen Mördern (oder Vergewaltigern) umfassen diese eine phallische Waffensymbolik,

\footnotetext{
${ }^{8}$ Linda Williams bezieht ihren Begriff der body genres wiederum von Carol J. Clover, die diesen in ihrem Aufsatz „Her Body, Himself: Gender in the Slasher Film“, ihrer Teilstudie zum Slasherfilm, von 1987 verwendet.
} 
Opfertypen und Schockmomente (ebd. 21-64), an denen Clover das transgressive Spiel mit heteronormativen Gender-Schemata und die genderspezifische Cross-Identifikation des vornehmlich männlichen Publikums mit dem Final Girl beschreibt. Diese sind analytisch auf die Sequels und Bates Motel anwendbar, wobei in den Sequels die Elemente der Waffen, Opfer und Schockmomente einbezogen werden, während Bates Motel hinsichtlich der von Clover in Rückbindung an Butlers Gender-Begriff konzipierten Gendersemantik des Horrors untersucht wird. Denn insbesondere die Slasher- und Rape-Revenge-Filme lösen nach Clover biologisch determinierte Geschlechtervorstellungen und heterosexuelle Gendernormen in einer queeren Lektüre des ,female victim-hero complex“ (ebd. 19) auf:

In both cases, the gender of the ,victim " part of the story (the rape sequence in the rape-revenge film, the flight-and-pursuit sequence in the slasher) overrides the gender of the ,hero" part of the story. [...] I proposed that the willingness of the slasher film to re-present the traditionally male-hero as an anatomical female suggests that at least one traditionally heroic act, triumphant self-rescue, is no longer strictly gendered masculine. The rape-revenge film is a similar case, only more so; it is not just triumphant self-rescue in the final moments of the film that the woman achieves, but calculated, lengthy, and violent revenge of a sort that would do Rambo proud. (Paradoxically, it is the experience of being brutally raped that makes a ,man' of a woman.) What I am suggesting, once again, is that rape-revenge-films too operate on the basis of one-sex body, the maleness or femaleness of which is performatively determined by the social gendering of the acts it undergoes or undertakes. (Ebd. $158 \mathrm{f}$.)

Clovers und Williams' Konzepte bleiben lange Ausnahmen innerhalb genretheoretischer und genderbezogener Diskurse, wie Christine Gledhill in der Einführung zu Gender Meets Genre in Postwar Cinemas 2012 festhält:

Genre and gender representation, two key areas of Film Studies, have generated challenging theories and debate. However, bar some notable exceptions, these concepts rarely intersect. Studies of gender representation and sexed spectatorship largely subsume genre into narrative and visual organization. Studies of genre too often assume gender as a relatively unproblematic component of specific generic worlds. (1)

Auch im deutschsprachigen Diskurs bilden ausführlichere Betrachtungen des interdependenten Verhältnisses von Genre und Gender, wie es Andrea B. Braidt in ihrem Konzept des „Film-Genus“ schließlich entwirft, Ergebnisse jüngerer Diskussionen (u. a. Liebrand; Liebrand/Steiner). Dabei weisen die Konzepte neben der etymologischen Gemeinsamkeit - beide stammen vom lateinischen ,genus‘ ab - auch eine forschungsgeschichtliche Ähnlichkeit auf (I. Schneider 
16). Analog zur Genretheorie wird innerhalb der Betrachtung von Geschlechtlichkeiten sukzessive von der Idee einer inneren Wesenseigenschaft Abstand genommen, um im Anschluss an Judith Butlers Thesen von einer grundsätzlichen kulturellen Konstruktion von Geschlecht und Geschlechtlichkeit auszugehen. Männlichkeit und Weiblichkeit bilden performative Ergebnisse sozialer Handlungen und medialer Inszenierungen, die biologisch nicht als determiniert zu setzen sind, sondern anti-essentialistisch zu konzipieren sind. Aus diesen Similaritäten fügen sich Genreformen und Genderkonfigurationen zu einem sich gegenseitig befruchtenden Verhältnispaar zusammen, in dem sich Genres immer auch als über Gender-Konfigurationen formierbar und veränderbar begreifen lassen und Gender-Formationen ebenso Genre-Konstellationen beschrieben werden (Blaseio 44), wie dies in der Analyse von Bates Motel herausgearbeitet wird.

In den letzten Jahren sind zudem intermediale Ansätze und Diskussionen zur Hybridität hervorzuheben, die wesentliche Neuerungen in die Genretheoriedebatten eingebracht und den Blick für intermediale Perspektiven weiter geöffnet haben, wie unter anderem Jason Mittells intermedialer Cluster-Ansatz (,A Cultural Approach“ und „Genre and Television“), der Genres innerhalb vielfältiger Wechselverhältnisse verortet:

We need to look beyond the text as the locus for genre and instead locate genres within the complex interrelations among texts, industries, audiences, and historical contexts. The boundaries between texts and the cultural practices that constitute them (primarily production and reception) are too shifting and fluid to be reified. Texts exist only through their production and reception, so we cannot make the boundary between texts and their material cultural contexts absolute. Genres transect these boundaries, with production, distribution, promotion, and reception practices all working to categorize media texts into genres. (Mittell, „Cultural Approach“ 7)

Intertextualität gehört zum Grundgerüst genretheoretischer Konzeptualisierungen. Als eine Art gemeinsamer Nenner sieht Scheinpflug die Funktion vieler Genrediskurse darin begründet, dass sie zur Bezeichnung und Perspektivierung von intertextuellen Strukturen genutzt werden (Scheinpflug, „Formelkino“ 65). Da sich Genreformationen nicht nur in einem Medium wiederfinden, sondern sich vielmehr medienübergreifend entwickeln, ist der intertextuellen Struktur eine Intermedialität hinzuzufügen. Aus der Genrepraxis bieten sich zahlreiche Beispiele für eine solche produktive, intermediale Genrebetrachtung an. Im Bereich des Western sind es diverse kulturelle Praktiken, die dessen mediale Ikonografie prägen: Literarische Werke über Abenteuergeschichten in der Zeit des Wilden Westens, Groschenhefte (dime-novels) über Buffalo Bill, populäre Wild West Shows in den USA und Völkerschauen in Europa entwerfen und etablieren 
bereits eine Medialität des Western, die vom Medium Kino schließlich übernommen sowie weiterentwickelt und inzwischen auch erfolgreich in Videospielen fortgeführt wird (Kirsten, „Western remediated“), wo verschiedene Adaptionen filmischer Genremuster zu beobachten sind (u. a. Mosel und Klein). Auch der Film noir lässt sich auf Genretraditionen in der Literatur zurückführen, wie beispielsweise die hardboiled novels, und in Fernsehserien, Comic-Reihen oder Videospielen in Form noir-typischer Stile wiederfinden. Ähnliches gilt für das Kriminalgenre, in dem Detektive Kriminalfälle in allen Medien ermitteln und mit Sherlock Holmes ein überaus prominentes Beispiel für eine medienübergreifende Genrefigur auszumachen ist, aber auch für den Horrorfilm, dessen Monster und haunted houses Überbleibsel der gothic novel-Erzählungen bilden. Intermediale Sichtweisen ermöglichen es, generische Beziehungen als Mediengeschichten zu erarbeiten, was nach Hickethier nicht nur die bestehende Differenz zwischen Kinofilm und Fernsehfilm überwindet, sondern auch Kontinuitäten zwischen den Medien verdeutlicht (,Genretheorie“ 89).

Neben diesen intermedialen Perspektiven (u. a. Bartosch; Bleicher, „Genre und Fernsehen“; Rauscher und Vogt) entwerfen Genreuntersuchungen vermehrt Genres als hybride Phänomene. Im Zuge globaler Austauschbewegungen und medialer Konvergenzphänomene, die sich nicht erst seit der Digitalisierung entwickeln, stellen für Ritzer und Schulze Genres als ,,sites of conflicting discourses and representations" (18) besondere Aushandlungsfelder für eine hybride und transnationale Popkultur dar. ${ }^{9}$ Durch die Zusammenführung genretheoretischer Konzepte mit Ansätzen der Post Colonial Studies lassen hierbei die allgegenwärtige ,Internationalität', ,Transnationalität " oder ,Hybridität " von Genrereferenzen in einem neuen Licht erscheinen, das der bisherigen hegemonialen Perspektive auf Hollywood entgegenarbeitet. Denn Mischungen aus lokalen und globalen Genreformen finden sich sowohl im sogenannten ,Minor Cinema', wo sie aber keineswegs nur eine Reaktion auf Hollywoods Marktdominanz darstellen, als auch in der US-amerikanischen Filmindustrie, in der durch das europäische Filmexil und nicht zuletzt als Verkaufsstrategie zum Zwecke der Markterweiterung ebenso externe Genreeinflüsse zu beobachten sind (Kirsten, „Film noir“). Doch nicht nur die Intermedialität und Hybridität generischer Formen bilden aktuelle Herausforderungen der Genretheorie.

Vor dem Hintergrund aktueller medialer Phänomene wie dem Web 2.0, der Medienkonvergenz, dem transmedia storytelling und gesamtgesellschaftlicher Vorgänge wie der Globalisierung gewinnt auch das filmwissenschaftlich geprägte Genrekonzept

${ }^{9}$ Zum transnationalen Kino siehe Bergfelder. 
eine intermediale und interdisziplinäre Dimension. Angesichts der vielen multimodalen und medienübergreifenden Entwicklungen, die auch in der digitalen und vernetzten Medienkultur der Gegenwart durch audiovisuelle Bestandteile gekennzeichnet und von einem filmkulturellen Genrebewusstsein beeinflusst sind, sollte das filmwissenschaftliche Genrekonzept auch in Zukunft angewendet, diskutiert und erweitert werden. (Kuhn et al. 32; Herv.i.O.)

Mit der zunehmenden Verbreitung von mobilen Endgeräten, Streaming-Diensten, Small Screens und der Omnipräsenz medialer Inhalte sind Genres nicht nur als transkulturelles und hybrides Aushandlungsfeld relevant. Sie gewinnen ebenso für die mediale Zirkulation und multimediale Verwebung von Inhalten und Formen an Brisanz und stellen bisherige Genrekonzepte vor neue Herausforderungen. Gerade im Zwischenbereich von Herstellung und Lektüre finden sich distributionsseitig zentrale Elemente, die metagenerische Funktionen erfüllen und auffällige Genremarkierungen enthalten. Diese Bedeutung von Werbematerialien und Vermarktungspraktiken findet in der Genretheorie meist nur randseitig Erwähnung. Die meisten Genretheorien richten sich vor allem an den Produktionen als zentralem Gegenstandsbereich aus, auch wenn sie das spezifische Produktionsumfeld als Kontext einbeziehen. Etwa werden produktionsseitige Bezeichnungspraktiken für historische Genreuntersuchungen analysiert (Staiger, „Hybrid or Inbred“; Altman „Film/Genre“ 49-68) oder rezeptionsseitige Diskurse abseits der Produktionen betrachtet wie bei Neale, der sich den Filmkritiken der 1930er Jahren zuwendet, um die semantische Verschiebung des Genrekonzepts des Melodrams zu beleuchten (,Melo Talks“). Doch der Fokus der meisten genretheoretischen Studien bleibt weiterhin auf die Produktionen und deren textuelle Merkmale ausgerichtet, auch wenn Genremerkmale hierbei nicht mehr als textimmanent verstanden werden. Dass aber die Werbematerialien und Vermarktungsstrategien wesentlich zur Konstituierung von Genrezusammenhängen beitragen, zeigen Hediger und Vonderau in ihrer Betrachtung von Genrebegriffen und -signalen in Werbematerialien bereits in Ansätzen auf. An anderer Stelle betont auch Neale die Bedeutung von Werbematerialien und Filmkritik für die Herausbildung von ,generic images“ bei Filmen (,Questions of Genre“ 182). Innerhalb genretheoretischer Betrachtungen stellen diese Ansätze allerdings noch Ausnahmen dar. Für die vorliegende Studie, insbesondere für Psycho und Bates Motel, bilden diese Materialien und Praktiken allerdings ebenso wichtige Genrediskurse wie die Diskurse der Filmkritik und Wissenschaft aus. Aus diesem Grund werden sie gleichwertig zu den iterativen Genreebenen analysiert.

Die Theoriegeschichte zu Genrekonzeptualisierungen zeigt, wie sich Genres auf unterschiedlicheren Ebenen (Einzeltheorie, allgemeine Genretheorie, Einzelanalyse) mit verschiedenen Theoriebegriffen beschreiben lassen. Für die 
genrehistorische Untersuchung von Psycho, den Sequels und Bates Motel kann damit kein einheitliches Analysemodell entwickelt werden, das die Komplexität der historischen und kulturellen Kontexte, Diskurse und Ebenen adäquat erfasst und im Vorhinein abstrahiert. Da die historischen Analysen von Psycho, den Sequels und Bates Motel auf den Ebenen der Genreproduktion und des Genrekonzepts operieren, sind sie sowohl innerhalb des ersten Feldes der Einzeltheorie zu verorten als auch Einzelanalysen zuzuordnen. Während Psycho als historisches Fallbeispiel in seinen Genrediskursen untersucht wird, werden die Sequels in Bezug zum Genrekonzept des Horror- und Slasherfilms als Genregruppierung analysiert, wohingegen Bates Motel in seinem distributionsseitigen Genrediskurs sowie in Bezug zu Psycho und den Sequels als genrespezifische Serienproduktion beleuchtet wird. Ausgerichtet an dieser materialnahen Genreuntersuchung nimmt zwar auch die vorliegende Studie eine Metaperspektive auf allgemeine Fragen der Genretheorie ein, indem sie ausgehend von den generischen Markierungen, Iterationen und Semantiken die dynamischen Genremuster als Genresignaturen konzipiert. Sie entwirft daraus jedoch kein geschlossenes Theoriemodell. Vielmehr demonstrieren die folgenden Ausführungen die produktive Kombinationsfähigkeit theoretischer Ansätze und methodischer Zugänge, die trotz unterschiedlicher fachhistorischer Kontexte in einem analytisch ausgerichteten Theorie- und Methodendesign zusammengeführt werden können: Nicht nur werden ,etablierte ' historische Ansätze der Genretheorie (Altman, Neale, Schweinitz) mit ,neueren ' phänomenologischen Theorien zu Gender und Genre (Clover, Williams) zusammengebracht, sondern auch mit ,älteren “ poststrukturalistischen (mitunter abseits dezidierter Genretheorien) Zugängen (Derrida, Deleuze und Foucault) verknüpft und als überaus kompatibel zueinander aufgezeigt. Welche Ansätze, Theorien und Zugänge hierbei für welche Untersuchungsebenen und welches Fallbeispiel herangezogen werden, wird im Folgenden deutlich, wenn entlang der zentralen Prämissen der Diskursivität und Historizität die Diskurse, Dynamiken und Ebenen beleuchtet werden, die Genres allgemein und die spezifischen Genreausprägungen des Untersuchungskorpus besonders kennzeichnen.

\subsection{Genres als diskursive Größen}

Genres sind allgegenwärtig. Sie bestimmen Herstellungsprozesse, Distributionswege und Vermarktungspraktiken, organisieren Programmübersichten von Kino, Fernsehen und Streamingdiensten und durchziehen kulturkritischwissenschaftliche Diskussionen und den filmgeschichtlichen Kanon ebenso wie 
Publikumsreaktionen und Fandebatten. Angesichts dieser Nutzungsbreite besteht in der Genretheorie allgemein Einigkeit darüber, dass Genres sich durch einen multidimensionalen und diskursiven Charakter auszeichnen: „All agree that genre is a multi-dimensional phenomenon and that its dimensions centrally include systems of expectation, categories, labels and names, discourses, texts and corpuses of texts, and the conventions that govern them all.“ (Neale, „Genre and Hollywood" 25 f.) Auch wenn in dieser Breite und Vielfalt an Genrebegriffen und Verwendungskontexten die Schwierigkeit besteht, eine übergreifende Genretheorie zu entwickeln, liegt darin auch die produktive Kraft von Genres begründet. Denn wie Sarah Berry-Flint herausstellt: ,The variety of contexts and uses for generic labels is important because it indicates the provisional nature of such categories. In practical terms, genres are vehicles for the circulation of films in industrial, critical, and popular discourses." (BerryFlint 26) So arbeiten beispielsweise historische Genretheorien die dynamischen Verhältnisse von Genremerkmalen auf, während kognitivistische Ansätze die rezeptionsseitige Steuerung und Verständigung durch Genres untersuchen. Angesichts dieser Vielschichtigkeit des Genrekonzepts bedient sich die Arbeit eines Diskurs-Begriffs, der Michel Foucault entlehnt ist. Mit Foucault lassen sich die Diskurse jeweils als ,eine Menge von Aussagen“ beschreiben, die ,zur selben diskursiven Formation gehören“ und ,durch und durch historisch“ (170) sind. Daran anschließend können fünf Diskurse identifiziert werden, ,die systematisch die Gegenstände bilden, von denen sie sprechen“ (74): Produktion, Publikum, Distribution/Marketing und Kritik/Wissenschaft, wobei diese nur auf einer analytischen Ebene voneinander zu trennen sind. Vor dem Hintergrund dieser Diskurse beschränkt sich das Untersuchungskorpus auf distributionsseitige Materialien und kulturkritisch-wissenschaftliche Diskurse, ohne aber die Herstellungs- und Rezeptionsseiten ganz auszublenden. In der Praxis unterliegen die Diskurse zirkulären Austauschverhältnissen, denn Mitglieder einer Produktion sowie filmkritisches und wissenschaftliches Personal sind immer auch als (Fach-) Publikum zu verstehen, dessen Genrewissen nicht nur auf Produktionserfahrungen oder theoretisch-analytischen Auseinandersetzungen beruht, sondern dessen Seherfahrungen ebenso einer medialen Sozialisation entspringen. Die verfolgte Perspektive setzt sich anschließend mit dem Verhältnis zwischen Genreproduktion und Genrekonzept als produktivem Differenzverhältnis von Genres auseinander. Das Kapitel diskutiert anschließend poststrukturalistische Ansätze der Markierung und Konventionalisierung, die genrespezifische Wahrnehmungen steuern und ein Genrebewusstsein zusammenhalten. Schließlich rücken Vorgänge der Wiederholung und Variation in den Fokus, die als sich wechselseitig bedingende Verfahren die generischen Diskursivierungen des Untersuchungskorpus durchziehen. 


\subsubsection{Distributionsseitige Materialien und kritische Diskurse}

Genres formen nicht einfach nur Gruppenzusammenhänge zwischen Produktionen, die ähnliche Merkmale aufweisen, sondern sie bilden Bezeichnungsgrößen für komplex angelegte, multidimensionale Phänomene, die sich ,als ,Instanzen des Gleichgewichts ' auf veränderte Dispositionen des kulturellen Umfelds einstellen“ (Schweinitz, 107). Was als ,Genre" verstanden wird, entsteht erst innerhalb der soziokulturellen, institutionellen und produktionstechnischen Konstellationen des jeweiligen Entstehungskontextes einer Produktion. Während des Herstellungsprozesses ermöglichen Genres Mitgliedern eines Produktionsteams einen Modus der Kommunikation, um sich über spezifische Produkteigenschaften auszutauschen sowie konkrete Produktionsentscheidungen zu treffen. Diese Verständigungen nehmen neben der grundsätzlichen, distributionsseitigen Genrezuteilung im Sinne Altmans auch eine aktive Rolle bei der Produktion ein, indem vor dem Hintergrund von Genrekonzepten oder erfolgreichen Vorgängerproduktionen an entsprechend bekannte Muster angeschlossen wird.

Per Definition gehören alle Filme - zumindest in den Verleihkategorien - Genres an, aber nur bestimmte Filme sind auf ein Genre hin produziert und werden aufgrund eines speziellen Genre-Typus konsumiert. Der Genrebegriff - wenn er Verleihoder Klassifizierungszwecken dient - bezeichnet Filmgenres. Hingegen redet man von Genrefilmen, wenn der Begriff des Genres eine aktivere Rolle in der Produktion und im Konsum spielt. (,Film und Genre“ 254)

Diese produktionsseitigen Verständigungs- und Herstellungsvorgänge zielen zwar auf eine Gestaltung und öffentliche Sichtbarkeit von Produktionen als Genreproduktion, insbesondere innerhalb der Vermarktung; von dieser kann aber nicht vice versa auf die vorausgehenden, produktionsseitigen Genrediskurse geschlossen werden. Vielmehr sind sie von den rezeptionsseitigen Lektüren zu unterscheiden und können erst über explizite Produktionsforschungen erschlossen werden, wie sie die Production Studies diskutieren (u. a. Caldwell, Mayer, Szcepanik/Vonderau). Dennoch lassen sich über die Produktionskontexte allgemeine Rückschlüsse auf die Rolle von Genres innerhalb von Herstellungsabläufen ziehen, wie es insbesondere Staiger für die serielle Arbeitsweise des klassischen Hollywoodsystems als Produktionsmodus aufzeigt (Bordwell et al.; Staiger, „Hybred and Inbred“ 208 ff.). ${ }^{10}$ Da auch der vorliegende Untersuchungskorpus

${ }^{10}$ Gegenüber diesem Produktionsdiskurs, der die industriellen Zusammenhängen vom Produkt her beschreibt, stellen die neueren Ansätze der Production Studies den Prozess der Herstellung selbst in den Fokus der Analyse (Vonderau, ,Theorien zur Produktion“ 14 f.). 
im Kontext von Hollywood steht, werden die Produktionen in den Analysen über eine historisch-ökonomische Perspektive kontextualisiert, wie bereits einführend skizziert, aber innerhalb der Analysekapitel hinsichtlich der genrespezifischen Produktionsumstände detaillierter ausgeführt. Diese so vorgenommenen Kontextualisierungen können zwar den produktionsseitigen Herstellungsprozess nicht dezidiert beleuchten, aber sie verorten die Produktionen innerhalb ihrer historischen Genrepraktiken, die auch für rezeptionsseitige Diskurse einen Kontextrahmen bereithalten.

Innerhalb von rezeptionsseitigen Diskursen erfüllen Genres ebenso wichtige Verständigungsfunktionen. Nicht nur schaffen sie für das Publikum Orientierung innerhalb diverser Programmangebote; sie erleichtern auch die individuelle Auswahl und kollektive Entscheidungsprozesse, weil sie Vorlieben, Erfahrungen und Erwartungen strukturieren und einen Kommunikationsmodus über potentiell zu Sehendes herstellen. ${ }^{11}$ Zwar verlaufen viele Diskussionen und Fanpraktiken inzwischen über digitale Medien innerhalb eines öffentlichen Raums, der für Genrestudien zugänglich wäre. Da dies aber nicht für die historischen Kontexte von Psycho (1960) und für die Sequels (1980er Jahre) gilt, beziehungsweise historiografische Archivleistungen erfordert, wird diese Ebene aus Gründen der Einheitlichkeit auch für Bates Motel ausgespart. ${ }^{12}$ Stattdessen wird einerseits von historischen Publikumsgrößen in einem abstrakten Sinn ausgegangen, die über Vermarktungspraktiken und Werbematerialien als Zielgruppen adressiert werden und über die historische Kontextualisierung in verschiedene Publika spezifiziert werden können. Anderseits wird innerhalb der Analysen der Fallbeispiele von ,Zuschauenden' als analytische Kategorie ausgegangen, die in den medialen Texten als Blickposition konstruiert wird.

Wie sich nach diesem ersten Blick bereits abzeichnet, nehmen die distributionsseitigen Diskurse eine entscheidende Schnittstellenposition ein, über die sowohl das Publikum als auch die Produktionsebene mitgeführt werden, auch wenn diese in ihren spezifischen Genrediskursen nicht dezidiert analysiert werden. Die distributionsseitigen Diskurse sind überdies aus mehreren Gründen von besonderem Genreinteresse. Einerseits stehen sie in einer medialen Öffentlichkeit, für die sie explizit hergestellt werden. Anderseits übernehmen sie für einzelne Produktionen ankündigende Funktionen, weil sie darauf zielen, die jeweilige

\footnotetext{
${ }^{11}$ Zur Verständigungsfunktion von Genres siehe Casetti und Keppler/Seel.

${ }^{12}$ Zugleich bedarf es für die rezeptionsseitigen Diskurse des Publikums von Bates Motel ethnografische Zugänge und Ansätze der Nethnografie, um aussagekräftige Ergebnisse zu generieren, die hier nicht zu leisten sind. Zur Nethnografie siehe Einwächter.
} 
generische Spezifik der einzelnen Filme im Umfeld des entsprechenden Angebotsspektrums von Kino, Fernsehen oder Streaming-Diensten herauszustellen. Neben Stars, Plot, Schauwerten und Grad des Realismus bilden auch Genres symbolische Vermarktungselemente, um über konkrete Genrebezeichnungen oder genrespezifische Rhetoriken und Bildlichkeiten auf Plakaten, Aushängen oder in Trailern und Webseiten die Produktionen genrespezifisch zu ,labeln“ und damit zielgruppenorientiert zu vermarkten. Die Werbematerialien bilden aufgrund der genrespezifischen Gestaltungsmöglichkeiten und zentralen diskursiven Stellung zwischen Produktions- und Publikumsbereichen einen wichtigen Genrediskurs innerhalb der vorliegenden Untersuchung. Statt der üblichen Konzeptualisierung als „Paratexte“, werden die Werbematerialien hier aber als ,Materialien“ verstanden, die keine den Produktionen nur ,äußerlichen' Beiwerke darstellen, sondern einen Diskurs im Sinne Foucaults formieren. Ein kurzer Blick auf den Paratext-Begriff verdeutlicht, warum dieses Konzept nicht herangezogen wird.

Mit Paratexten beschreibt Gérard Genette eine problematische ,äußere Kontur' des Textes, die letztlich alles, was außerhalb von diesem liegt, paratextuell erscheinen lässt. ${ }^{13}$ Kritisch ist nach Stanitzek, dass zwischen dem ,eigentlichen ${ }^{\text {* }}$ Text und dem Beiwerk nicht zweifelsfrei unterschieden werden kann und Elemente aus dem Text herausgelöst und abgetrennt werden, um sie wiederum auf den Text als Ganzes zu beziehen. Der somit entblößte ,nackte Text' wird zum Oppositum der Paratexte erklärt, wobei er weder konkretisierbar noch vorstellbar ist, sondern nur ein regulativer Horizont für die Argumentation bildet (,Texte, Paratexte" 6). Das Konzept zielt einzig auf die Konkretisation des literarischen Werkes als „Einheit der Differenz von Text und Paratext“ (ebd. 8). Für das vorliegende Untersuchungskorpus, insbesondere die Fallanalysen zu Psycho und Bates Motel, bilden diese Materialien aber nicht ,nur' Randphänome eines darüber hinaus bloß vage auszumachenden Haupttextes, sondern vielmehr in einem poststrukturalistischen Sinn diskursive Instanzen, die ,systematisch die Gegenstände bilden, von denen sie sprechen“ (Foucault 74) und den ,generischen Text"

${ }^{13}$ Auf Genette geht eine erste Konzeptualisierung zurück: „Der Paratext ist jenes Beiwerk, durch das ein Text zum Buch wird und als solches vor die Leser und, allgemeiner, vor die Öffentlichkeit tritt. Dabei handelt es sich um [...] eine Schwelle oder [...] eine»unbestimmte Zone « zwischen innen und außen, die selbst wieder keine feste Grenze nach innen (zum Text) und nach außen (zum Diskurs der Welt über den Text) aufweist [...].“ (,Paratexte“ 10). Ansätze zu dieser Theorie finden sich bereits in Palimpsestes (11 ff.). Film-, fernseh- und medienwissenschaftliche Forschungen übertragen ab Anfang der 2000er Jahre das Konzept auf audiovisuelle und digitale Gegenstände (siehe u. a. Böhnke, Böhnke et al., Gray, Gwóźdź, Kreimeier/Stanitzek und Nitsche). Eine ausführliche Kritik von Genettes Paratextbegriff findet sich bei Stanitzek (,Texte, Paratexte“). 
als semantische Einheit - im Zusammenspiel mit den anderen Diskursen und in Bezug zu semantischen Genrekonzepten - erst erzeugen.

Von Werbematerialien geht aber nicht nur eine generische Diskursivität aus, sondern auch eine generische Materialität, denn sie verhelfen Genres angesichts der zwischen Genreproduktion und Genrekonzept herrschenden strukturellen Lücke, die nachfolgend als konstitutiver Bestandteil von Genres erläutert wird, zu einer konkreten Sichtbarkeit in Form von „generic images“ (Neale, „Questions“ 182). Neale weist auf die Bedeutung von ,institutional discourses“ (ebd. 185) hin, die filmindustrielle (Werbe-)Materialien und Filmkritiken umfassen, weil diese erst die Existenz von Genres bezeugen und Evidenzen herstellen: „In the public sphere, the institutional discourses are of central importance. Testimony to the existence of genres and evidence of their properties, is to be found primarily there." (Ebd.) Vor allem distributionsseitig und vermarktungstechnisch eingesetzte Plakate, Trailer, Aushangfotos, Werbeanzeigen etc. verbinden konkrete Genrebezeichnungen und generische Ankündigungsrhetoriken mit konventionalisierten Genreikonografien, sodass Produktionen eine bestimmte ,Generizität` erhalten.

Genre is, of course, an important ingredient in any film's narrative image. The indication of relevant generic characteristic is therefore one of the most important functions that advertisements, stills, reviews, and posters perform. Reviews nearly always contain terms indicative of a film's generic status, while posters usually offer verbal generic (and hyperbolic) description - ,The Greatest War Picture Ever Made' - as anchorage for the generic iconography in pictorial form. (Ebd. 181)

Die Idee der Zeugenschaft, wie sie Neale für die Diskurse der Filmkritik und Werbematerialien aufmacht, ist für das Untersuchungskorpus, insbesondere für Psycho, dahingehend wichtig, dass sie einen Zugriff auf den damaligen ,praktischen' Genrediskurs bereithalten, wie unter anderem die Filmkritik von Peter John Dyer illustriert: „Psycho contains one of the bloodiest murders ever filmed, he then asks this spirited actress to sustain the daylight panic of a secretary in love who robbed her firm of $\$ 40,000$.“ (195) Aber auch Trailer und Plakat verkünden: „Alfred Hitchcock's Greatest Shocker Psycho - A Paramount Picture“ und „A New - And Altogether Different - Screen Excitement“.

Da das Untersuchungskorpus also nicht nur auf die textuelle Analyse der Produktionen von Psycho, den Sequels und Bates Motel zielt, sondern ebenso die Werbematerialien als Genrediskurse untersucht, wird im weiteren Verlauf die Bezeichnung als ,Materialien“ benutzt, um eine methodische Gleichwertigkeit zu markieren. Durch den Wegfall des ,Werbe'-zusatzes wird die Werbefunktion und Hierarchisierung dieser Materialien unter den zu bewerbenden Produktionen 
aufgebrochen. In digitalen Medienkulturen zirkulieren sowohl Medienproduktionen als auch Materialien in einer nach Paech und Schröter formalen Kontinuität als ,Film“ (11) beziehungsweise ,Serie“ intermedial zwischen Kino(wieder-) aufführungen, Fernsehausstrahlungen, DVD-Veröffentlichungen und digitalen Streamingdiensten. ${ }^{14}$ In Bezug auf die mediale Diversität der Materialien ist allerdings ein reiner intermedialer Ansatz weniger geeignet, sodass zusätzlich auf eine Multimedialität zurückgegriffen wird, die, wie Ruchatz formuliert, ,,das Zusammengehen ,ganzer" Medien meint" (16) und die ,klassischen" Materialien wie Plakate und Trailer ebenso innerhalb eines Medienverbunds zusammenbringt wie digitale Angebote auf DVD- und Blu-ray-Editionen, YouTube-Kanälen und Q\&A-Formaten, ohne diese in ihrer Medienspezifik aufzulösen. Anderseits wird in Bezug auf die Materialien neben einem diskursanalytischen Genreansatz und einer multimedialen Überblicksperspektive auch eine transmediale Perspektive eingenommen, die generisch konventionalisierte Genreikonografien als „medienunspezifische ,Wanderphänomene““ (Rajewsky 12) begreift, wobei der medienspezifische Kontexte für die Funktion der generischen Wirkung zwar noch wichtig ist, aber nicht mehr ein medialer Ursprung ausgemacht werden muss.

Zusätzlich zu diesem distributionsseitigen Diskurs der Materialien stehen auch kulturkritische Diskurse im Fokus, wobei diese getrennt von den Materialien betrachtet werden und nicht wie bei Neale gemeinsam als ,institutional discourses" erfasst werden. Die kulturkritischen Diskurse ermöglichen eine generische Perspektivierung der Produktionen, die eine ,theoretisch-analytische“ Genauigkeit verfolgt. Anderseits wird die Filmkritik in ihren Genrediskursen von den Konzeptualisierungen der Genretheorie unterschieden. Auch wenn die kulturkritischen wie wissenschaftlichen Diskurse Genres für diverse Fragen der Zuordnung, Entwicklung und Bewertung benutzen (Hickethier, „Genretheorie“ 63), bilden sie unterschiedliche diskursive Formationen aus. Während Genres in Kritiken stärker für normative Wertmaßstäbe genutzt werden, wie die Filmkritiken zu Psycho sehr deutlich zeigen, ermöglichen Theoriekonzepte ,Aussagen «mittlerer Reichweite> zwischen der Makroebene (Theorien des Films) und der Mikroebene (Analyse einzelner Filme)“ (Hickethier, „Genretheorie“ 62) und die Wiedergabe der historischen Entwicklung von Genres.

Dieser erste Blick auf Genres als diskursive Größen verdeutlicht vor allem die Relevanz der distributionsseitigen Materialien, die zusammen mit den Diskursen der Filmkritik einen Zugriff auf produktionsspezifische Genrediskurse bereithalten. Diese können aber erst über eine historisch-ökonomische Perspektive

\footnotetext{
${ }^{14}$ Eine Übersicht zur Intermedialität als Konzept von Medienbeziehungen findet sich bei Ruchatz.
} 
(bei Psycho und Bates Motel) und einen genresemantischen Bezugsrahmen (bei den Sequels und Bates Motel) in ihren Genresemantiken analysiert werden. Denn erst die historische Kontextualisierung der Diskurse zeigt auf, inwiefern eine einzelne Produktion generisch ,gelabelt ${ }^{\text {' }}$ ist oder eine Gruppe an Produktionen generisch zusammenhängt. Während die Materialien und Filmkritiken zu Psycho erst in ihren Verbindungen zu damaligen Genrekonventionen des Horrorfilms und zu Hitchcocks, Thrill and Suspense'-Kino als Genrediskurse lesbar werden, können die Sequels und Bates Motel erst über den Rückgriff auf das semantische Konzept des Slasher- und Rape-Revenge-Films in ihren Iterationen genrespezifisch beschrieben werden. Indem Genrediskurse, ob in Form von Filmkritiken und Materialien einer Genreproduktion oder in Form von Iterationen einer Genregruppierung, jeweils zwischen den Ebenen von Genrekonzept und Genreproduktion vermitteln, bilden sie im Foucault'schen Sinn ihre Gegenstände ,systematisch * zu semantischen Einheiten. Anders formuliert heißt dies für die historischen Analysen, dass die Genrediskurse (Psycho und Bates Motel) und generischseriellen Diskursivierungen (Sequels und Bates Motel) zwar in Bezug auf die inter-/textuellen/seriellen Strukturen der Produktionen operieren, aber in ihrer Generizität durch „sets of cultural conventions“ (Tudor, „Genre“ 7), von außen“ konstruiert werden. Die Textdimension der Beispiele wird hierbei keineswegs überflüssig; sie bildet vielmehr in ihrer generischen Verfasstheit ein Ergebnis der diskursiven Verhältnisse, die im Sinne einer offen zu verstehenden Intertextualität wie Diskursivität von Genres den generischen Text (Genreproduktion) beziehungsweise den generisch-seriellen Intertext (Genregruppierung) als semantische Einheiten erst nachträglich hervorbringen. Inwiefern dieses wechselseitige Verhältnis von Genrekonzept und Genreproduktion ein zentrales Differenzverhältnis für Genres bildet, aber auch ein strukturierendes Moment der Analysen, wird im Folgenden genauer beleuchtet, bevor die Textdimension von Genres weiter ausgeführt wird.

\subsubsection{Genreproduktion und Genrekonzept}

Das Verhältnis von Genreproduktion und Genrekonzept lässt sich anhand einiger methodischer Hürden verdeutlichen, die im analytischen Umgang mit Genres immer wieder betont werden. Im Gegensatz zu der im alltäglichen Umgang mit Einzelgenres und Genreproduktionen aufscheinenden Evidenz von Genres weisen theoretisch-analytische Systematisierungen Zirkularitäten und Lücken auf. Viele Genrebegriffe, Gattungsbezeichnungen und Formatbegriffe stammen nach Hißnauer ursprünglich aus Diskursen der Praxis, wo sie nicht einheitlich entwickelt wurden, aber erhalten in wissenschaftlichen Auseinandersetzungen meist 
den Status analytischer Kategorien (143). Angesichts der fehlenden einheitlichen Systematisierung, der pragmatischen Nutzung und der verschiedenen Abstraktionsniveaus gestalten sich sowohl genaue und verlässliche Definitionen einzelner Genres als auch eindeutige Klassifikationen zu einem unmöglichen Unterfangen, wie Ansätze seit den 1980er Jahren betonen. Genres reproduzieren sich nicht einfach entlang einer festen spezifischen Struktur, noch liefern Genrekonzepte einen fixierten Code. Anders formuliert bilden Genres keine einheitlichen Genrekonzepte aus, die immer wieder passgenau und identisch in Genreproduktionen angewandt werden können, ebenso wenig wie Genreproduktionen einem festen Muster folgen. Dies stellt die Korpusbildung von Genreanalysen vor ein methodisches Problem, da die Auswahl an Produktionen für genreanalytische Untersuchungen von Einzelgenres bereits eine inhärente Genregruppierung mit sich führt. Diese methodologische Zirkularität liegt nach Hickethier in generischen Gruppenbildungsprozessen an sich begründet, da Filme, die gemeinsame Erzählstrukturen, Themen und Motive aufweisen, sich zu einer Gruppe zusammenfügen lassen, aus der die gemeinsamen Merkmale schließlich verallgemeinert und zu Prinzipien des Genres erhoben werden. Für die einzelnen Filme werden diese Prinzipien wiederum als normativer Maßstab angesetzt, um über deren Zugehörigkeit oder Ausschluss zu entscheiden („Genretheorie“ 150 f). Bereits Anfang der 1970er Jahre hat Tudor diese methodische Hürde als empirisches Dilemma der Genreforschung identifiziert, weil von generischen Gemeinsamkeiten und Ähnlichkeiten ausgegangen wird, die durch die Untersuchung eigentlich erst zu belegen sind („Genre“ 5). Als Abstraktionen aus einer Gruppe von Filmen, die zu einem Genre gerechnet werden, gehen Genres nicht in nur einem Film aus dieser Gruppe auf; aus der Gruppe kann wiederum kein Film, als Teil dieser Abstraktion, ein vollständiges Beispiel für das Genre liefern. So geht weder Psycho vollständig im Konzept des Horrorfilms oder des Thrillers auf, auch wenn er innerhalb beider Konzepte einen historischen Wendepunkt markiert, noch kann der Slasherfilm vollständig in den Sequels abgebildet werden, auch wenn die Fortsetzungen als eine generische Zykluseinheit für die Analyse auf der Ebene des Genrekonzepts herangezogen werden. Ebenso lässt sich Bates Motel nicht nur einem Genrekonzept zuordnen, was allein schon dem Umstand geschuldet ist, dass Serien erzählerische Ressourcen zur Verfügung stehen, um Figurenkonstellationen und Handlungsstränge generisch auszudehnen.

Aufgrund dieser Problematik, Genres umfänglich an nur einer Produktion festzumachen, als auch der Schwierigkeit, Genres empirisch vollständig über alle zu einem Genre zu rechnenden Produktionen wiederzugeben, ohne Zirkelschlüsse zu liefern, bleibt zwischen dem einzelnen Film und der Idee vom Genre als Korpus stets eine strukturelle Lücke bestehen (Hagener 1). Diese ist für das 
Verhältnis von Genreproduktionen und Genrekonzepten kennzeichnend. Indem aber Genres als diskursive Größe konzipiert werden, die keiner vorausgehenden festen Struktur und keinen einheitlichen Merkmalen folgen, sondern jeweils ihre Merkmale in Diskursen prozessieren, lässt sich die strukturelle Lücke als ein konstitutiver Bestandteil von Genres begreifen, durch den sich Genreproduktion und Genrekonzept stetig wechselseitig konstituieren müssen, weil keine Seite als feste Größe vorausgesetzt werden kann. In den Diskursivierungen kann diese strukturelle Lücke von Genres nie vollständig überwunden werden, sondern sie wird als Bezugsverhältnis permanent aufrechterhalten. Das Verhältnis gerinnt so zu einem grundsätzlichen, produktiven Differenzmoment, das Genres in der steten Fortschreibung aber nicht nur stabilisiert (Hagener 19), sondern im kulturellen Gedächtnis lebendig hält, sodass sie für das Publikum gleichermaßen evident erscheinen wie sie für Analysen erkenntnisreich bleiben. In der Differenz nehmen konkrete Genrebezeichnungen eine Art Vermittlungsposition zwischen Genreproduktion und Genrekonzept ein. Deren latente Unschärfe und Variabilität ist hierbei ,notwendig für das Funktionieren der Genrebegriffe im kommunikativen Gebrauch“. (Hickethier, „Genretheorie“ 65). In Anwendung auf Produktionen setzen Genrebezeichnungen nach Derrida eine erste generische Markierung, mit der eine Zugehörigkeit zum semantischen Konzept hergestellt wird. In Bezug auf Genrekonzepte evozieren Genrebezeichnungen Merkmale, die von mehreren Produktionen geteilt werden und von Tudor als Sets kultureller Konventionen beschrieben werden: „Genre notions - except in the arbitrary definition - are not critics' classifications made for special purposes; they are sets of cultural conventions.“ („Genre“ 7; Herv.i.O.) Um diese Markierungen und Konventionen im Kontext einer poststrukturalistischen ,Textdimension" geht es im Folgenden.

\subsubsection{Genremarkierungen im Kontext eines Genrebewusstseins}

Texte erscheinen nicht unabhängig von generischen Kontexten. Auch wenn ein Text nicht explizit generisch bezeichnet ist, erfolgt seine Wahrnehmung doch stets über Genres, indem sich der Text selbst über Markierungen (,re-marks“) ausweist: ,this re-mark - ever possible for every text, for every corpus of traces - is absolutely necessary for and constitutive of what we call art, poetry, or literature.“ (Derrida, „The Law“ 64) Nicht nur können somit mehrere Genrezugehörigkeiten vorgenommen werden. Die Art und Weisen, wie diese sich ergeben, fallen vielfältig aus und umfassen nicht zwangsläufig Titelbezeichnungen, wie es Derrida beispielsweise für literarische Untertitel wie ,Roman' oder ,Drama“ 
anzeigt. Denn abseits dieser Titelpraxis können in jedem ästhetischen Korpus generische Spuren (,traits“) identifiziert und Genrezugehörigkeiten (,belonging“) identifiziert werden. Die Markierungen weisen allerdings den Umstand auf, selbst nicht genrezugehörig zu sein, weil sich der Text zwar selbst markiert, aber darin zugleich die Markierung verschwindet.

(A) text cannot belong to no genre, it cannot be without or less a genre. Every text participates in one or several genres, there is no genreless text; there is always a genre and genres, yet such participation never amounts to belonging. And not because of an abundant overflowing or a free, anarchic, and unclassifiable productivity, but because of the trait of participation itself, because of the effect of the code and of the generic mark. Making genre its mark, a text demarcates itself. If remarks of belonging belong without belonging, participate without belonging, then genre-designations cannot be simply part of the corpus. (,The Law“ 65)

Statt also Genres einfach nur , anzugehören', sind Texte auf vielfache Art und Weise generisch markiert, wodurch sie spezifische Erwartungen und Vorstellungen wecken, aber auch an mehreren kulturellen Codes und Konventionen ,teilnehmen“ (,participate“), ohne dass diese Markierungen selbst zu genrehaften Eigenschaften gerinnen. Weder gehen Markierungen vollständig in den Texten oder im Korpus auf, noch sind sie rein äußerliche Erscheinungen: ,it does not, in whole or in part, take part in the corpus whose denomination it nonetheless imparts. Nor is it simply extraneous to the corpus." (Ebd. 65) Genres bewegen sich vielmehr genau an der Schnittstelle und Grenze zwischen der Genreproduktion, die als ,einzelner Text` über Genrebezeichnungen und textuelle Merkmale generisch markiert ist, und dem Genrekonzept, an dessen kulturellen Konventionen der Text über seine Markierungen teilhat. So wird nach Derrida sowohl der ,generische Text' generiert als auch das Genrekonzept als Korpus zusammengehalten, ohne dass einer/s von beiden vollständige, abgeschlossene Einheiten ausbildet. Mit dieser poststrukturalistischen Betrachtung von Genres geht eine grundsätzliche Offenheit von Text und Korpus als ,the limitless field of general textuality" (63) einher, wie sie auch in den Analysen deutlich wird: Während die Genrediskurse von Psycho und Bates Motel einen ,generischen Serien-/Text" konstruieren, erzeugen die iterativen Muster in den Sequels und Bates Motel einen , generisch-seriellen Intertext ${ }^{\star}$. Diese Inter-/Serien-/Texte entstehen aber nur, weil sie in Bezug zu kulturellen Genrekonventionen diskursiviert und semantisiert werden. Mit Schweinitz' Genrebewusstsein bietet sich dafür ein Erklärungsansatz, der die scheinbare ,innere' Konsistenz von Genrezusammenhängen auf die Konventionalisierung von Prototypen zurückführt. Bevor auf den Prototypen-Ansatz eingegangen wird, soll kurz die von Genres ausgehende Konsistenz entlang von 
Ordnungsverhältnissen kritisch diskutiert werden. Zwar bewirken Genres übergeordnete Ordnungszusammenhänge; sie sind darin aber keineswegs konsistent. Genres unterliegen sowohl einem Moment der Konstruktivität als auch einem Moment der Normativität, denn sie ziehen Grenzen ein und bestehen darauf, diese selbst gezogenen Grenzen nicht zu verletzen.

As soon as the word, genre' is sounded, as soon as it is heard, as soon as one attempts to conceive it, a limit is drawn. And when a limit is established, norms and interdictions are not far behind: ,Do, ',Do not' says ,genre, ' the word ,genre, 'the figure, the voice, or the law of genre. (,,The Law“ 56)

Diese ,Ordnungen“ werden in der Genretheorie häufig als generic systems konzipiert, deren dynamische Zusammenhänge zwischen einzelnen Genres oft als „open systems“ verstanden werden (Cohen 210). Genresysteme verbinden ,in terms of broad shared principles" einzelne Genres miteinander, wobei sie von der Ebene der Genreproduktion zu unterscheiden sind: „Generic systems and genres do not exist in the way in which individual films exist, but rather are abstractions based partly, though not entirely, upon individual films and operating in a different logical universe." (Ryall 329) Dieses weite Verständnis eines Genresystems beruht auf einem unspezifischen Systembegriff und weist keine Nähe zur Systemtheorie von Luhmann auf. Vielmehr wird unter dem Genresystem ein Kontextkonzept verstanden, das für die einzelne Genreproduktion einen übergreifenden intertextuellen Produktions- und Rezeptionsrahmen bietet (ebd.). Kuhn et al. differenzieren diesen , systemischen Kontext' in spezifische Produktionskontexte, wie das amerikanische Genresystem, und in medienspezifische Genresysteme, wie unter anderem das Videospiel oder auch den Film (20 f.). Letztlich werden mit dem ,Genresystem“ kontextuelle Zusammenhänge bezeichnet, ohne aber den systemischen Fragen nach der Existenz, Konstitution und Operationsverfahren eines ,Genresystems“ nachzugehen. Welche Komplexität mit einer Konzeptualisierung von Genre als systematischem Regulativ von Produktion und Rezeption einhergeht, verdeutlicht Hickethier:

Wird das Genre nicht nur als eine Kategorie zur Beschreibung sprachlicher Handlungen, sondern darüber hinaus als systematisches Regulativ in Produktions- und Rezeptionsprozessen verstanden, also unter medienästhetischen, produktions- und rezeptionstheoretischen Aspekten betrachtet, ist zu klären: (1) welche Normen und Regelhaftigkeiten in ihm eingeschrieben sind, die Produktion und Rezeption beeinflussen; (2) wie die Struktur des Genrewissens beschaffen ist (die Bestandteile des Genres und die >Grammatik < ihrer Verknüpfungen) und wie es systematisiert (Genresystematik) und gespeichert wird (Genregedächtnis) und unter welchen Bedingungen 
sich dieses Genrewissen verändert; (3) in welchem Verhältnis Genrewissen und Genrepraxis zueinander stehen; (4) zu welchem Zweck Genres entstehen und welche Funktionen sie ausüben als Teil der Produktionsbedingungen von Medienprodukten, in der Rezeption von Filmen und als Ordnungssysteme innerhalb der gesellschaftlichen Kommunikation. (,Genretheorie“ 70).

Angesichts dieser systemischen Komplexität von Genres scheint der Versuch, ein übergreifendes Genresystem zu entwickeln, ebenso aussichtslos wie die Definition einer einheitlichen Makrostruktur für ein Genrekonzept, ${ }^{15}$ beziehungsweise stellt sich als ähnlich langwierig heraus wie die Entstehung von Luhmanns Systemtheorie.

Genres stellen vielmehr widersprüchliche Ordnungen auf, in denen sich Genrekonzepte überschneiden und Genreproduktionen plurale Markierungen aufweisen, die sie an mehreren generischen Konventionen teilnehmen lassen. Die zwischen Produktionen suggerierte Ähnlichkeit hält einer genauen Betrachtung nicht stand. Dieser , widersprüchliche Anschein' gründet auf einer ,inneren' Konsistenz von Genrezusammenhängen, die Schweinitz über ,die das Bewusstsein dominierende Rolle von Prototypen“ (111) erklärt und die gerade für Psycho von entscheidender Bedeutung ist. Im kognitionspsychologischen ,Erfahrungsrealismus' von Lakoff, auf den sich Schweinitz bezieht, werden Kategorien entlang von menschlicher Erfahrung und Imagination automatisch und unbewusst als eher ,inhomogene ' Gruppen gebildet, die keiner generellen Regel oder festen Grenzen folgen, sondern vielmehr durch einen, zentralen Fall` zusammengehalten werden. Dieser ,zentrale Fall ' entspricht aber keinem Muster, sondern besteht in mehreren Variationen, sogenannten Subkategorien, die sich um Konventionen gruppieren. Prototypen bilden hierbei exemplarische Variationen. Sie dominieren das Bewusstsein, die Erfahrung, weil sie „mit dem ,zentralen Fall“ korrespondieren und gegebenenfalls der Exemplifizierung dienen“ (ebd.). Da auch für Genres einzelne Produktionen paradigmatisch sind und diese wie Prototypen funktionieren, liegt für Schweinitz der Schritt nah, ein an Lakoffs Prototyp-Ansatz orientiertes Genrebewusstsein zu konzipieren. Ausgehend von einer radialen Assoziationsstruktur werden mit einem Genre ein bis zwei Prototypen assoziiert, die die Vorstellung und Erwartung dieses Genres dominieren und hinter denen die restliche Menge an zugehörigen Genreproduktionen verschwindet. Hierbei greifen für Prototypen sowohl persönliche Vorlieben als auch kulturelle Normierungen, die

${ }^{15}$ Siehe Schweinitz (110). Die von Hickethier identifizierten Fragestellungen könnten über Ansätze der Systemtheorie gelöst werden und so eine systemtheoretische Genretheorie aufbauen. Solch ein systemtheoretischer ,Überbau' könnte auch die strukturelle Lücke von Genres auflösen. Dies wäre allerdings eine komplett andere Arbeit. 
Produktionen als ,Klassiker des Genres ‘ wiederholt aufbereiten, sodass sie sowohl bei der Produktion als auch der Rezeption als konventionalisierte Prototypen wahrgenommen werden (ebd.).

Erst das Genrebewusstsein verleiht dem ,Genre-Code“ als Faktor innerhalb des filmkulturellen Diskurses lebendige Existenz. Erst das praktisch wirksame Genrebewusstsein sorgt dafür, dass das Konzept ,Genre“ sowohl bei der Filmproduktion als auch bei der Rezeption als Orientierungsgröße funktioniert. (Ebd. 113)

Dieser Ansatz eines Genrebewusstseins wendet sich von der Vorstellung formaler Textklassen ab, die ein Korpus aus regelkonformen Invarianten aufweisen, und einer Betrachtung konkreter Assoziationsmomente zu, die Genrezusammenhänge in ihrer Dynamik und Variation jenseits einheitlicher Kategorisierungsversuche begreift. Das birgt für die Analysen drei wichtige Momente: (1) Ausgehend von der heutigen prototypischen Funktion von Psycho, die er insbesondere für den Horrorfilm und Psycho-Thriller aufweist, werden auf der Ebene des Genrekonzepts die Genrediskurse retrospektiv dahingehend beleuchtet, wie Psycho, der 1960 als Randphänomen des Genres wahrgenommen wird, zu einer paradigmatischen Stellung in der Genregeschichte kommt, und welchen Einfluss Produktionsund Rezeptionskontext, Genregeschichte und Theoriegeschichte haben. (2) Auf der Ebene des Genrekonzepts bedeutet dies wiederum, dass die Sequels als ein exklusiver Genrekorpus des Slasherfilms dahingehend diskursiviert werden, wie sie mit Psycho nicht nur einen im Kanon der Filmkunst etablierten Film aufgreifen, sondern eben jenen Film fortführen, verändern und in einen generisch-seriellen Intertext überführen, der nachfolgend von Clover als ,ancestor" beschrieben wird. ${ }^{16}$ (3) Für die integrative Betrachtung der Ebenen von Genreproduktion und Genrekonzept heißt dies schließlich, dass Bates Motel sowohl vor dem Hintergrund von Psycho's genrehistorischem Klassikerstatus zu betrachten ist als auch in Bezug zu den generisch-seriellen Zusammenhängen von Psycho und den Sequels gesetzt wird. Inwiefern in all diesen Diskursivierungen, wie auch für Genres generell, Vorgänge der Wiederholung und Variation eine zentrale Rolle einnehmen, wird im Folgenden beleuchtet.

\footnotetext{
${ }^{16}$ In einer früheren Version der Teilstudie zum Slasherfilm bezeichnet Clover bereits 1987 Psycho als ,immediate ancestor“ („Her Body“ 192), aber auch diese erstmalige Zuschreibung erfolgt nach Psycho III.
} 


\subsubsection{Wiederholung und Differenz}

Für Genres wird immer wieder betont, dass sie sowohl Wiederholungen als auch Variationen unterliegen, wie unter anderem bei Barry Keith Grant: ,genre movies are those commercial films that, through repetition and variation, tell familiar stories with familiar characters in familiar situations." (XVII) Innerhalb einer Produktion lassen sich die generischen Markierungen als derart wiederholte Muster beschreiben, denn die Markierung gibt sich letztlich als ,Wiederholung' zu erkennen, um damit als ,bekannt' identifizierbar zu werden. Inwiefern in der Wiederholung bereits ein Moment der Variation enthalten ist, macht Gilles Deleuze deutlich, der in der Wiederholung eine ,unaustauschbare, unersetzbare Singularität“ sieht (,Differenz“ 15), die sich stets in Bezug zu etwas Singulärem und damit Einzigartigem versteht, aber darin zu nichts anderem ähnlich oder äquivalent ist. Auch wenn sich wiederholende Gesten äußerliche Erscheinungen sind, wie es Deleuze am Beispiel von Feiertagen ausführt, so verkehrt sich die Wiederholung in ihrem Bezug zur Potenz, ,indem sie sich nach innen stülpt“ (ebd. 15 f.). Von der Gewohnheit, die ebenso Abläufe wiederholt, aber als angenommene Verhaltensweise konstante Intentionen oder gleiche Kontexte voraussetzt und darin keine echte Wiederholung darstellt, grenzt Deleuze die „Wiederholung als Universalität des Singulären“ ab (ebd. 16).

Die Wiederholung ist Sache des Humors und der Ironie; sie ist ihrer Natur nach Überschreitung, Ausnahme und behauptet immer eine Singularität gegen die dem Gesetz unterworfenen Besonderheiten ein Universales gegen die Allgemeinheiten, die als Gesetz gelten. (Ebd. 20)

Dabei bildet die Wiederholung in Deleuzes Denken mit der Differenz eine Einheit, weil sie sich gegenseitig bedingen wie wechselseitig konstituieren und darüber als prozesshafte Größen in Erscheinung treten.

Diese beiden Untersuchungen haben sich von selbst miteinander verschränkt, weil sich diese Begriffe einer reinen Differenz und einer komplexen Wiederholung unter allen Umständen zu vereinigen und zu verschmelzen schienen. Die permanente Divergenz und Dezentrierung der Differenz ist eng mit einer Verschiebung und einer Verkleidung in der Wiederholung verbunden. (Ebd. 12; Herv.i.O.)

In Deleuzes Arbeit ist dabei die Gegenlektüre anderer Denker eine Methode, die auf einer wechselseitigen Wiederholung und Differenzbildung beruht, indem nicht nur andere Texte aufgegriffen werden, sondern sich andere Texte im Feld der Philosophiegeschichte zu einem Text formen, in dem alle miteinander in Relation 
stehen (Ott 29). Dieses Verfahren arbeitet aktiv mit seinem Vorgängermaterial, indem es Taktiken ,,der langsamen Um- und Überschreibung des Ausgangstextes, der Verlängerung und Transformation seiner Begriffe, der Freilegung von Subtexten im Text" (ebd. 26) anwendet. Diese verlaufen selbst zirkulär; sie kreisen um Ideen, Gedanken, Bilder, transportieren und denken diese weiter, sodass in den reziproken Wiederholungsverfahren zugleich Differenzierungen stattfinden. Mit jeder neuen unter einem Genre geführten Genreproduktion verschiebt sich auch bei Genres das zugehörige semantische Konzept, denn keine Produktion gleicht der vorherigen. Um als genrespezifische Merkmale erkannt zu werden, so zeigen die bisherigen Ausführungen, sind sie davon abhängig, wiederholt und differenziert zu werden. In dieser Abhängigkeit bilden Genres Effekt und Ursache zugleich, denn Genres gehen zwar Produktionen logisch voraus, folgen aber zugleich faktisch nach.

Jeder Film bezieht sich auf Genre-Konventionen, schreibt sie aber gleichzeitig um, modifiziert und konstruiert sie. Das Genre (von dem wir doch eigentlich annehmen, dass es dem Film vorgängig ist), ist also immer ein Effekt jener Filme, in denen es sich ausdrückt/konkretisiert/dokumentiert. Wir haben es also mit der Schwierigkeit zu tun, dass das Genre nicht Film ist, aber uns nur im Film begegnet: Das Genre geht dem Film (logisch) voraus und ist doch (faktisch) sein Effekt. (Liebrand/Steiner 2)

Die Signifikanz und zugleich latente Varianz von Genres beruht auf nachträglich gezogenen Differenzen. Anders formuliert heißt das, dass Genres nicht einfach das Immergleiche wiederholen, sondern erst in der Differenz verdeutlichen können, was wiederholt wird, womit sie immer schon in einem Verhältnis zu einem anderen stehen, das zuvor bereits wiederholt worden ist. Diese sich wechselseitig bedingenden Prozesse der Wiederholung und Differenz durchziehen die generischen Diskursivierungen der Filmkritik, Film- wie Genretheorien zu Psycho, die Materialien von Psycho und Bates Motel sowie die generisch-seriellen Iterationen in den Sequels und Bates Motel. Ziel der Analysen ist es, vor dem Hintergrund kultureller Genrekonventionen, die über die Kontextualisierung der Produktionen und Genrepraktiken sowie über semantische Genrekonzepte skizziert werden, die spezifischen Differenzverfahren herauszuarbeiten, die Genres sowohl zu stets aktuellen, generischen Serien-/Texten“ als auch zu dynamischen, generischseriellen Intertexten“ gestalten. Auch wenn Psycho den Anfang der historischen Analysen markiert, so gilt für das Untersuchungskorpus wie Genres allgemein, dass weder ein vorgängiges ,erstes“ generisches Objekt, auf das eine generische Linie zurückzuführen ist, noch Ur-Genres auszumachen sind, die außerhalb einer Historizität bestehen und dabei noch eine generische Definitionshoheit 
besitzen: ,there are no arch-genres that can totally escape historicity while preserving a generic definition.“ (Derrida, „The Law“ 62; Herv.i.O.) Um diese Prämisse der Historizität von Genres geht es im Folgenden.

\subsection{Genres als historische Phänomene}

Genres transformieren sich stetig. Denn nicht nur werden Genres in unterschiedlichen Diskursen benutzt; mit jeder Verwendung geht wie oben ausgeführt auch eine latente Modifikation einher, die sich auf verschiedenen Ebenen beobachten lässt: Neben dem textuellen Gegenstandsfeld, bei dem keine Genreproduktion mit der vorherigen identisch ist, und dem semantischen Konzept, das durch jede neue zugehörige Produktion etwas hinzugefügt bekommt, unterliegen auch die Genrebezeichnungen einer historischen Dynamik und weisen Veränderungen, Kombinationen und Neubildungen auf. Dabei fügen sich die historischen Wandlungen von Genres in das obige komplexe Konstrukt aus Herstellungsprozessen, Publikumserwartungen, Vermarktungsstrategien und kritischen Diskursen ein, die ebenso Veränderungen unterliegen. Dies bedeutet, dass an Genres - so die Ausgangsthese - interne wie externe Veränderungen greifen, was Kuhn et al. als eine „filmhistorische Prozessualität“ darstellen (16). Wie diese historische Komplexität von Genres wiederum beschreibbar gemacht werden kann und für die historischen Analysen von Psycho, den Sequels und Bates Motel zu operationalisieren ist, steht im Fokus der folgenden Ausführungen. Dabei wird zuerst der Herausbildung von Genrebezeichnungen nachgegangen, die als historische Phänomene erste wichtige Beobachtungen über die Historizität von Genres erlauben, bevor die Anwendbarkeit historischer Schemata am Beispiel von Hickethiers Phasenmodell diskutiert wird. Der allgemeinen Perspektive dieses Modells wird anschließend der historische Zyklen-Ansatz von Grindon gegenübergestellt, der von iterativen Mustern ausgeht und sowohl für eine gesamtgenerische Betrachtung des Untersuchungskorpus als auch der Fallanalysen geeigneter ist.

\subsubsection{Genrebezeichnungen}

In den ersten Jahren des frühen Kinos werden die Filme meist nur über Länge und Thema, wie „Kampfbilder“, aber kaum über präzise Genrebezeichnungen vertrieben (Altman, „Film und Genre“ 253). Insbesondere Verleihkataloge verdeutlichen diese frühe Bezeichnungspraxis, die von den heutigen etablierten Genres wie Horrorfilmen, Western oder Kriegsfilm noch weit entfernt ist und beispielhaft über 
zwei Kataloglisten veranschaulicht werden kann: 1905 listet die Kleine Optical Company ihr Angebot nach Story (darin noch unterteilt in historical, dramatic und narrative), Comic, Mysterious, Scenic und Personalities, während die American Mutoscope and Biograph Company 1902 ihre Filme aufteilt in die Kategorien „Comedy Views, Sports and Pastime Views, Military Views, Railroad Views, Scenic Views, Views of Notable Personages, Miscellaneous Views, Trick Pictures, Marine Views, Children's Pictures, Fire and Patrol Views, Pan-American Exposition Views, Vaudeville Views, and Parade Pictures“ (Neale, „Questions of Genre“ 187). Schließlich werden ab Beginn des 20. Jahrhunderts zunehmend Genrebezeichnungen eingesetzt, um das Angebot der vielteiligen Nummernprogramme der Varietétheater und Jahrmarktskinos anzukündigen (Hickethier, „Genretheorie“ 74). Diese ersten Genrebezeichnungen setzen sich meist aus zwei Wörtern zusammen: einem Adjektiv und einem Substantiv. Während ersteres besondere Merkmale hervorhebt, umfasst zweiteres einen allgemeinen, bereits bekannten Genrebegriff, wie Altman am Western und Musical veranschaulicht. Diese Genres gehen terminologisch auf adjektivische Substitute zurück, die als ,Western chase film“, „Western melodramas“ oder auch ,,musical comedy“, „,musical romance“ mit bereits etablierten Genrebezeichnungen aus dem Revue-Theater, Drama und der Literatur kombiniert werden („Film/Genre“ 52 f.). Die sich so herausbildenden generischen Bezeichnungen bilden nach Staiger neben Plot, Schauwert, Grad des Realismus und Stars nur eines der vielen ,Verkaufsargumente', um das Angebot an Filmen spezifisch zu bewerben. Hierfür ist die Einführung eines geregelten Verleihsystems ab 1909 von entscheidender Bedeutung, da Kinobetreibende nun rechtzeitiger und verbindlicher erfahren, wann sie neue Filme zeigen und über welche Verkaufsargumente sie diese bewerben können. Ab 1915 richtet sich die Filmwerbung nicht nur zunehmend an dem neuen Format des Langspielfilms aus, das sich schnell als Standard durchsetzt und zur Hauptattraktion des Kinoprogramms wird, sondern letztlich werden alle Filme gesondert angekündigt (,Waren anpreisen“ 23). Das führt dazu, ,dass die Filmwerbung zwar Produktdifferenzierung leistet, aber von der gängigen Praxis anderer Industriezweige abweicht, indem sie jedem Einzelprodukt individuelle Identität verleiht" (ebd. 24).

Bereits am frühen Kino lassen sich damit wichtige historische Beobachtungen über Genres festhalten: Nicht nur werden Genres als ,kulturindustrielles Prinzip“ (Schweinitz, 101) bereits vor 1910 genutzt, vielmehr entspringt ihre Bezeichnung einem Vertriebs- wie Werbebedürfnis. Dies schließt an die oben herausgestellte analytische Schnittstellenposition distributionsseitiger Diskurse und die zuvor resümierte diskursive Konstituierung von Genres an, bei der kein vorgängiges ,erstes‘ generisches Objekt auszumachen ist. Zugleich wird deutlich, 
dass Genres im frühen Kino nicht einfach ,erfunden', sondern aus bereits etablierten Unterhaltungsformen des Theaters und der Literatur übernommen werden und sich damit medienübergreifend ausbilden.

Während der Studio-Ära gewinnen die spezifischen ,Besonderheiten ‘ der Filme schließlich derart an Bedeutung, dass immer häufiger der aus anderen Unterhaltungsbereichen übernommene generalisierende Teil wegfällt und ,eigene filmische' Genrebezeichnungen in substantivischer Form entstehen. Hierbei zeigt Altman nicht nur für amerikanische Filmgenres, sondern auch kulturhistorisch für die Komödie und Tragödie, wie sich Genres zu autonomen Kategorien ausbilden: „In each case the development of the stand-alone noun signals the liberation of the former adjective from its noun and the formation of a new category with its independent status.“ („Film/Genre“ 51) Mit der Emanzipation der Substitute filmischer Bezeichnungen wie ,Western melodramas' und ,musical romances“ zeichnet sich also die Herausbildung eigenständiger Genrekonzepte ab. Allerdings gehen nach Altman dieser Substantivierung wichtige Veränderungen auf der Ebene von Herstellung, Text und Rezeption voraus, bevor filmische Genrebezeichnungen eine vollständige Generifizierung erreichen. Einerseits verschiebt sich durch Standardisierung und Automatisierung der Herstellungsfokus von den generalisierenden Nomen auf die generischen Substitute, sodass der ,Westernoder auch ,Musical'-Anteil wichtiger wird als die Generalität von ,melodrama“ oder ,romance'. Anderseits übersteigen in den Filmen die Genremerkmale die reine adjektivische Namensgebung und verbinden sich mit den generellen Merkmalen derart zu generischen Spezifika, dass sich ,Western' nicht mehr nur auf den Wilden Westen bezieht, sondern ebenso melodramatische Handlungen und Figuren umfasst; oder auch ,Musical' nicht mehr nur in Bezug zum reinen Musikeinsatz steht, sondern zum Katalysator und zur Ausdrucksform der heterosexuellen Liebeshandlung wird. Beides steht in unmittelbarer Verbindung mit einer veränderten Wahrnehmung beim Publikum, das die Ähnlichkeiten zwischen den Filmen unter dieser filmischen Genrekategorie erkennt und Erwartungshaltungen entwickelt. Diese beeinflussen schließlich die generische Identifizierung und Bedeutungsgebung (ebd. 53). Genres resultieren nach Altman nicht nur aus dem produktionsseitigen Bedarf, die Herstellungsmethoden $\mathrm{zu}$ standardisieren und $\mathrm{zu}$ rationalisieren, um profitabler zu werden sowie das Absatzrisiko berechenbar zu machen. Vielmehr unterfüttert Altman die Standardisierungsthese mit einem veränderten Wahrnehmungsbewusstsein auf Seiten von Produktion und Rezeption. Die Handlungsmotive, Erzählmuster und Bildformen werden unter der entsprechenden , adjektivischen' Genrebezeichnung erst dann generisch, wenn sie bei der Produktion sowohl als erfolgreich und als wiederholungswürdig gelten als auch für das Publikum einen Wiedererkennungswert besitzen und Erwartungen 
schüren (ebd.). Anders formuliert heißt das, dass sich Genres als semantische Einheiten etablieren, wenn sie, wie bereits Schweinitz für das Genrebewusstsein unterstreicht, wiederholt zur Herstellung und zur Lektüre herangezogen werden. Diese von Altman beschriebenen Wandlungsprozesse gelten nicht nur für die amerikanische Kinoindustrie, sondern auch für europäische oder andere Filmkulturen, in denen sich angesichts des bereits im frühen Kino festzustellenden großen Produktionsumfangs zwangsläufig Genrebezeichnungen und Genrekonventionen entwickelt haben, die bis in die Gegenwart verlaufen.

Eine erste historische Dimension von Genres bildet also die terminologische Entwicklung von Genres. Entlang der veränderten Genrebezeichnungen wird deutlich, wie sich Genrekonzepte einzelner Filmgenres als semantische Einheiten bei der Produktion und Rezeption herausbilden, indem sie wiederholt auf Produktionen angewendet werden. Dies ist insofern für die historischen Analysen relevant, als dass konkrete Genrebezeichnungen zwar einen ersten Bezugspunkt für Genreproduktionen und Genrekonzepte erlauben, aber ebenso historische Phänomene bilden wie die Ebenen, die sie bezeichnen. Daher müssen die für Psycho, die Sequels und Bates Motel verwendeten Genrebegriffe ebenso vor dem Hintergrund ihres Entstehungs- und Produktionskontextes untersucht werden wie die Produktionen und Genrekonzepte. Denn einerseits können unter der gleichen Genrebezeichnung je nach historischem Kontext unterschiedliche semantische Konzepte subsumiert werden, wie Neale am Beispiel des Melodrams aufzeigt: Mit diesem Genre ist in den 1930er Jahren eine Spannungsorientierung verbunden, die mit der heutigen Auslegung als Genre pathetischer und romantischer Empfindsamkeit und exzessiver Sentimentalität, wie es unter anderem Williams beschreibt („Film Bodies“), nicht zusammenpasst. Die Semantik des Melodrams hat sich fast vollständig in ihr Gegenteil verkehrt: „The mark of these films is not pathos, romance, and domesticity but action, adventure, and thrills; not ,feminine " genres and the woman's films but war films, adventure films, horror films, and thrillers, genres traditionally thought of as, if anything, ,male.'“ (Neale, „Melo talks" 69) Dies findet sich auch noch in den Filmkritiken zu Psycho, in denen mit „old-fashioned melodramatics“ genau auf diese Spannungsgenerierung referiert wird, auch wenn sie dem Film flache Schockeffekte vorwerfen (Crowther 37). Anderseits wurden ältere Produktionen bereits Genrekonzepten zugeordnet, die als Bezeichnungs- und Semantisierungsoption zum Zeitpunkt der Entstehung der Produktionen noch gar nicht zur Verfügung standen und unter denen folglich die Produktionen auch nicht wahrgenommen werden konnten, wie es für The Great Train Robbery von Edwin S. Porter aus dem Jahr 1903 der Fall ist. Wie erneut Neale an anderer Stelle verdeutlicht, beschreiben genrehistorische Studien der 1960er Jahre den Film als ersten und einflussreichen Western (,QQuestions of 
Genre“ 186). ${ }^{17}$ Diese Wahrnehmung entspringt allerdings retrospektiven Lesarten, die den Film erst nachträglich unter diesem Genrekonzept diskursivieren. Der Erfolg und Einfluss des Films ist weniger vor dem Hintergrund des Westerngenres zu erklären, als vielmehr darauf zurückzuführen, „that is was the confluence of paradigms provided by melodrama, the chase film, the railway genre, and the crime film, rather that the western, that ensured the film's contemporary success" (ebd.). Dabei existiert der Western als spezifische Genrebezeichnung und semantisches Genrekonzept um 1903 weder für die Herstellung noch für das Publikum. Auch wenn die damals populären Wild West Shows, dime novels und zahlreichen Western-Abenteuergeschichten bereits den Western als Genre in anderen Unterhaltungsbereichen konventionalisieren: Erst zwischen 1903 und 1911 wird er als adjektivisches Substitut für viele Filme genutzt (Gallagher 300 f.). Damit bildet er für The Great Train Robbery nicht den generischen Rahmen (Neale, „Questions of Genre“" 186.). Er initiiert nachfolgend auch keine Westernfilme, sondern Kriminalfilme. Wie Neale mit Verweis auf Charles Musser zusätzlich herausstellt, ist der Erfolg des Films nicht nur auf die Popularität der Genres zurückzuführen, sondern auch auf mehrere kulturelle Trends und Strategien, die der Film geschickt aufzunehmen versteht:

One reason for The Great Train Robbery's popularity was its ability to incorporate so many trends, genres and strategies fundamental to the institution of cinema at that time. The film includes elements of both re-enactment of contemporary news events (the train hold up was modeled after recently reported crimes) and refers to a well-known stage melodrama by its title. Perhaps most importantly, The Great Train Robbery was part of a violent crime genre which had been imported from England a few months earlier. Porter was consciously working (and cinema patrons viewing) within a framework established by Sheffield Photo's Daring Daylight Burglary, British Gaumont/Walter Haggar's Desperate Poaching Affair [Affray] and R. W. Paul's Trailed by Bloodhounds. ... [Thus,] when initially released, The Great Train Robbery was not primarily perceived in the context of the Western. Its success did not encourage other Westerns but other films of crime - Lublin's Bold Bank Robbery [Jack Frawley, 1904] Paley and Steiner's [Avenging a Crime; Or,] Burned at the Stake [1904]. (Musser in ebd. 186 f.)

Die retrospektiv irreführende generische Diskursivierung von The Great Train Robbery als erster wichtiger Western schließt an Altmans terminologische Geschichte von Genres an und belegt, wie die Verwendung von Genrebezeichnungen und die Diskursivierung über Genrekonzepte von einem Genrebewusstsein auf Seiten von Herstellung und Rezeption abhängen. Neale spricht hierbei von

${ }^{17}$ Neale verweist hier auf Arbeiten von MacGowan (114) und Fenin/Everson (49). 
„generic regimes“, die verschiedene historische Zeiträume kennzeichnen und entlang derer Produktionen diskursiviert werden (ebd. 187). Für die historische Untersuchung von Genres heißt das:

Hence the importance of historicizing generic definitions and the parameters both of any single generic corpus and of any specific generic regime. For it is not that more elaborate definitions are impossible to provide, just that they are always historically relative and therefore historically specific. (Ebd. 191)

Wiederum für das Untersuchungskorpus bedeutet dies, mit den generischen Diskursivierungen kritisch umzugehen und die Bezeichnungen wie Semantiken nicht als Ergebnis des Entstehungskontextes anzunehmen, sondern als Ergebnis historischer Diskursivierungsprozesse zu betrachten. Diesen geht häufig ein anders gelagertes Genrebewusstsein voraus, das über Diskurse und Kontexte der damaligen Zeit zu erarbeiten ist, und zugleich folgt ihnen unter Umständen ein neues nach, sodass Produktionen diskursiven Genreverschiebungen unterliegen, wie im Fall von Psycho. Während sich Psycho's diskursiver Genrestatus vom Randphänomen zum Prototypen entwickelt, entstehen die Sequels und Bates Motel zwar unter dem film- und genrehistorischen Einfluss von Psycho, aber auch unter den neuen semantischen Genrekonzepten, die sich unter anderem durch Psycho verändert haben. Auf beides sind die Produktionen jedoch nicht zu reduzieren, denn in ihren jeweiligen Kontexten kommen wiederum neue populäre Medien- und Genreformen hinzu, wie nummerierte Sequels, Serien-reboots oder Slasher- und Mystery-Genres, die sich ebenso in den Produktionen niederschlagen. Aus diesem Grund eignet sich für das Untersuchungskorpus weniger eine Makroperspektive, wie sie das historische Phasenmodell von Hickethier liefert, sondern vielmehr der produktive Zyklen-Ansatz von Grindon.

\subsubsection{Makrogeschichte(n)}

Einteilungen der Genregeschichte in Phasen und Stufen nehmen eine evolutionäre Makroperspektive auf generische Entwicklungsvorgänge ein, die gegenüber der bisher erarbeiteten diskursiven und historischen Komplexität von Genres oft schemenhaft bleiben. Gemäß Hickethiers Modell entwickeln sich Genres ausgehend von einer Entstehungsphase (I) durch die schematische Verbindung von Sujets, Motiven und erzählerischen Mustern, mit denen zuvor experimentiert wurde, 
und durch die konkrete namentliche Benennung in kulturellen Diskursen. ${ }^{18}$ Auf Seiten von Produktion und Rezeption bildet sich so ein „konstitutives Bewusstsein“ („Genretheorie“ 71) heraus, das Hickethier Schweinitz’ Prototypen-Ansatz entlehnt. Dieses festigt und verankert sich über Prototypen in der anschließenden Phase der Stabilisierung (II). Während der Stabilisierung bildet sich eine erfolgreiche Gruppe an Filmen heraus, die in variierter Form ,typische“ Erzählmuster von Prototypen, wie es erneut Schweinitz konzipiert, aufgreifen und enge Ähnlichkeitsverhältnisse ausprägen. Die gelungene Wiedererkennung beim Publikum stellt dabei ,ein Resultat eines kulturellen Bedarfs nach bestimmten, in den Genres erzählten Geschichten und ein Ergebnis einer Gewöhnung an die dabei verwendeten Stereotypen“ (ebd. 72) dar. Die Phase der Erschöpfung (III) von Genres tritt anschließend durch die anhaltende Produktion von Varianten ein, wobei einzelne Zeitphasen zwar unterschiedliche Varianten eines Genres ausweisen, aber irgendwann alle möglichen Varianzen auserzählt haben, sodass häufig Parodien erzeugt werden. $\mathrm{Zu}$ dieser ,Auserzählung' kommt es durch kulturelle Veränderungen, die dazu führen, dass „Genres als erzählte und dargestellte kulturelle Ordnungssysteme ihre Funktionen für die Regulierung des jeweils aktuellen Selbstverständnisses verlieren“ (ebd. 73). Schließlich können in der letzten Phase der Neubildung (IV) durch die Integration von neuen Merkmalen Genres inhaltlich erweitert und mit anderen Genres gemischt werden, sodass Subgenres oder neue Genres entstehen. Auch die mediale Expansion trägt zu dieser Neuformulierung und intermedialen Erweiterung von Genres im Bereich von Fernsehen und seriellen Fiktionsformen bei. Für diese letzte Phase greift insbesondere die „Variabilität und Offenheit“ (ebd. 73) von Genres, die stetig weitere Transformationen und Neubildungen ermöglichen.

Mit diesem Phasenmodell trägt Hickethier der zunehmenden „Ausdifferenzierung in immer weitere Genres“ („Genretheorie“ 73) Rechnung und überführt die historische und diskursive Dynamik von Genres in ein allgemeines Schema der Genregeschichte, das in der Form einen generellen Überblick ermöglicht. Zwar bezieht Hickethier die vielzähligen internen wie externen Einflussfaktoren aus Prototypen, Genrebewusstsein, Varianten, kulturellen und medialen Kontexten ein, aber die historische Komplexität großer Zeitspannen wird auf eine modellhafte Evolutionsgeschichte reduziert. Kritisiert wird an diesen historischen

\footnotetext{
${ }^{18}$ Hickethiers Modell ähnelt dem vierteiligen Stufensystem von Thomas Schatz, das er 1981 in Bezug auf die Formenevolution von Henri Focillon und die von Christian Metz identifizierten Entwicklungsphasen des Western (vom Verfall des klassischen Western in den 1940er Jahren über den Superwestern der 1950er Jahre bis zum Italowestern) entwickelt, aber zu einem allgemeineren Modell der Genregeschichte generalisiert (,Hollywood Genres“ 3441).
} 
Modellen, wie sie nicht nur Hickethier, sondern auch Schatz und Metz aufstellen, dass sie eine evolutionistische Stabilität nur theoretisch aufrecht erhalten können und mitunter Filme und Praktiken außen vor bleiben, die in dieses Modell nicht passen, wie Tag Gallagher am Beispiel des Western belegt. Frühe Westernfilme weisen bereits 1909 in der ,Entstehungsphase ' des Genres eine „hyperconsciousness“ gegenüber ,(p)icturesque scenery, archetypal characters, dialectical story construction, long shots, close-ups, parallel editing, confrontial cross-cutting, montaged chases“ (301) auf, die vor 1895 längst in anderen Unterhaltungsbereichen konventionalisiert werden und damit der reinen Herausbildung widersprechen.

In fact, so popular were westerns during narrative cinema's formative years (1903 1911) that it may well be that, rather than the cinema invented the western, it was the western, already long existing in popular culture, that invented cinema. (300)

Nicht nur widerspricht die medienübergreifende Herausbildung von Filmgenres der Schematisierung; auch finden sich Genremischungen bereits in Phasen der ,Stabilisierung '. Schon im klassischen Hollywood gehören Genremischungen nicht nur zur bewussten Produktionspraxis, sondern werden ebenso als solche wahrgenommen, wie Janet Staiger nachweist. Filme werden vornehmlich entlang von konventionalisierten Doppelplotstrukturen produziert, um ein möglichst breites Publikum zu erreichen. Meist besteht eine davon aus einer heterosexuellen Liebesgeschichte, die das früh als vermarktungstechnisch wichtige Zielgruppe identifizierte weibliche Publikum anziehen soll („Hybrid or Inbred“ 208 f.). Gleichzeitig findet Staiger sowohl innerhalb produktionsseitiger Texte als auch in Filmkritiken der damaligen Zeit diverse Bezeichnungen und Beschreibungen, die auf doppelbödige und mehrdeutige Genrestrukturen in Filmen verweisen (ebd. 210), unter anderem auch auf Psycho's Doppelsemantik aus ,fairy tale with a detective story“ (Wollen nach ebd.). Schließlich können auch die grundsätzliche Offenheit und die strukturelle Variabilität von Genres nicht erst für die letzte Phase der ,Neubildung ' herangezogen werden, sondern gelten ebenso für frühe Formen, denn wie mit Neale und Gallagher deutlich wird, zeichnet sich die Popularität von Genres bereits im frühen Kino über die Fähigkeit aus, kulturelle Trends aufzunehmen.

Für das exklusive Untersuchungskorpus von Psycho, den Sequels und Bates Motel lässt sich Hickethiers Modell einer Makrogeschichte von Genres aus diesen Gründen nur bedingt heranziehen. Die vorliegende Studie zielt weniger auf eine allgemeine Genregeschichte, die überblicksartige Etappen modelliert, sondern 
visiert vielmehr eine ,kleine“ Genregeschichte über ihre spezifischen Genreausprägungen, jeweiligen Entstehungskontexte und generischen Diskursivierungen an. Zwar stehen Psycho, die Sequels und Bates Motel für unterschiedliche Genrevarianten einzelner Zeitphasen, wie es Hickethier für die ,Stabilisierung ‘ beschreibt und als Ursache für die ,Erschöpfung' ausmacht; sie resultieren aber zugleich aus medialen Expansionen, wie Hickethier es wiederum der ,Neubildung' zu Grunde legt. Zugleich gehen die Beispiele weder in einer allgemeinen Makrogeschichte auf, noch in einem einzelnen Genre, auch wenn das Horrorgenre ein zentraler Bezugspunkt bleibt. Denn schon Psycho lässt sich als ein Produkt des sich verändernden Mediensystems und eine Form der generischen Neubildung fassen, wie auch die Sequels und Bates Motel generischen Modernisierungstrends von Horror-Sequels und Prequel-Serien folgen. Statt einer Makroperspektive oder einer Geschichte des Horrorgenres nehmen die Analysen die Komplexität historischer und diskursiver Genrezusammenhänge in Blick, um die Produktionskontexte, Genrediskurse und Bezugsverfahren in materialnahen Fallanalysen herauszuarbeiten, ohne im Vorhinein von einer theoretischen Makrogeschichte oder einem Einzelgenre determiniert zu sein. Inwiefern dafür ein historischer Ansatz produktiver ist, der statt einer Makroperspektive die iterativen Muster von Genres entlang eines exklusiven Korpus in den Blick nimmt, wird im Folgenden erläutert.

\subsection{3 ,Exklusive' Genrezyklen}

Leger Grindon geht von einer Historizität der Iterationsmuster aus, über die Genres vor allem in ihrer diskontinuierlichen Entwicklung erfasst werden können. Als zentrale ,Akteure' im Wechselspiel mit externen wie internen Veränderungsprozessen lassen sich Iterationsmuster als Zyklen betrachten, die zu bestimmten Zeiten generische Subeinheiten generieren: „The cycle, a distinctive and more focused category, is a series of genre films produced during a limited period of time and linked by a dominant trend in their use of the genre's conventions." (44) Ähnlich hebt auch Altman die genrehistorische Bedeutung von Zyklen hervor, fügt sie aber mit den Substantivierungsprozessen der Genrebezeichnungen zu einem historischen Modell von Genres zusammen, in dem adjektivische Ergänzungen von Genrebezeichnungen erst subgenerische Zykluseinheiten bezeichnen, bevor sie selbst zu Substantiven und größeren Genreeinheiten werden, die wiederum neue adjektivische Ergänzungen und subgenerische Varianten nach sich ziehen (,Film/Genre“ 64 ff.). Das Zyklen-Konzept bezieht sich allgemein auf bestimmte Genrereihen von Studios, wie die Universal-Horrorfilme der 1940er 
Jahre, aber auch auf Kinoreihen wie die James Bond-Filme oder die Star WarsSequels oder Genreproduktionen wie Sergio Leones Italo-Western. Grindons Zyklusansatz zentriert statt der industriellen, figuren- oder namensbezogenen Generizität die generische Engführung von Iterationen, die innerhalb einer begrenzten Zeit übergreifend als Variationen auftauchen, wie die auf Halloween folgenden Slasherfilme (44 f.). Dies ist insofern für das Untersuchungskorpus ein produktiverer Ansatz, weil durch diese genrehistorisch engere Skalierung ein exklusives Korpus entlang dominanter intertextueller Muster gebildet wird, der sich in erster Linie an überschaubareren Zeitspannen orientiert und weniger an einheitlichen semantischen Genrekonzepten. Über die Iterationsmuster lassen sich die Produktionen generisch zusammenfassen, aber zugleich als subgenerische Ausprägungen in ihren spezifischen Entstehungskontexten analysieren. Grindon konzipiert Genres weniger als semantisch-intertextuelle Ganzheiten, sondern vielmehr als Ähnlichkeitsgruppierungen, die sich sowohl innerhalb eines Genres formieren als auch sich transgenerisch entwickeln und kulturelle Sets an Konventionen ausbilden (44), ähnlich wie es Tudor bereits beschreibt. Die verschiedenen Handlungsmuster, Figuren und Schauplätze, die Genres umfassen, gestalten dabei einen wiederkehrenden dramatischen Konflikt aus, wie beispielweise die Auswirkungen neuer Technologien im Science Fiction-Genre oder die American Frontier im Western (45). Nicht nur nehmen diese dramatischen Konflikte für Grindon eine wichtige kulturelle Rolle ein, weil sie soziale Problemstellungen der Gesellschaft behandeln; sie führen externe Einflüsse sowie interne Gestaltungsmerkmale zu einem generischen Vergnügungsangebot für das Publikum zusammen (47 ff.).

The generic crossroads allows the audience to enjoy the ritual satisfaction of overcoming social problems [...], while at the same time reinforcing traditional values that support the prevailing ideology. However, the pleasures offered by genre films rarely represent a viable solution for viewers, but rather, offer thrills that distract the audience from thinking through the real conflicts in their own experience. Genres thereby offer potentially subversive pleasures regulated by the cultural institutions constituted in and through the generic discourse. (49)

Gerade weil Grindon die genretypischen Konfliktsituationen nicht als feste Themen, ideologische Positionen oder Lösungsmuster ansieht, sondern als dynamische Vielfalt an iterativen Gestaltungsmöglichkeiten festmacht, die dem Publikum ein spezifisches Genrevergnügen bereitet, bilden Genres in seinem Konzept kulturell ,produktive Kräfte': ,The socially critical elements underlying the crossroads present genre as a more dynamic cultural force than either ritual celebration or the ideological entrapment models would suggest.“ (49) Dies berücksichtigt einen 
diskursiven und historischen Charakter von Genres, der historische Entwicklungen weniger als eine Evolutionsgeschichte erscheinen lässt, sondern vielmehr über diskontinuierliche Zyklen die dynamische Wandlungsfähigkeit von Genres beschreibbar macht. Innerhalb spezifischer zeithistorischer Kontexte lassen sich so Iterationsmuster als Akteure betrachten, die zwischen soziokulturellen Einflüssen und genreinternen Veränderungen ein Spannungsfeld erzeugen, das Genres vorantreibt: „The key link between external causes and internal changes is the social conflicts animating a genre." (52) $\mathrm{Zu}$ diesen externen Einflüssen und internen Veränderungen zählen für Grindon einerseits kommerziell erfolgreiche Produktionen, die den von Schweinitz beschriebenen prototypischen Charakter erhalten können und Genreneubildungen im Sinne Hickethiers initiieren können, anderseits aber auch filmindustrielle Faktoren wie Zensuren oder Altersfreigaben, technologische Entwicklungen wie Special Effects oder ökonomische Strukturen, die die Produktion und Rezeption von Genres beeinflussen, weil sie restriktive Vorgaben oder neue Möglichkeiten bereithalten, die auf eine industrielle Kompatibilität drängen. Hinzu kommen unterhaltungskulturelle Kontexte, deren populäre Phänomene und Trends ein breites Impulsfeld für Genreproduktionen bereithalten, wie in Bezug auf den Western und The Great Train Robbery von Gallagher und Neale betont wird, oder auch soziopolitische Ereignisse und Sensationen, die durch ihre mediale Präsenz Änderungen bekannter Muster und Konventionen hervorrufen (53 ff.).

Für das gesamte Untersuchungskorpus können nicht nur solche externen Faktoren aufgeschlüsselt werden, sondern auch interne Veränderungen, die Grindon auf das Wechselspiel von dominanten und subdominanten Genremerkmalen zurückführt und entlang von Umschichtungsvorgängen zu bestimmten Zeiten beobachtet: „Internal changes can be charted in the play between dominant and subordinate traits within a genre. The reshuffling of dominant and subordinate positions among the conventions in cycles is a key to tracing a genre's evolution." (55) Im Zuge von Ermüdungserscheinungen und Vorhersehbarkeiten bestimmter Muster können untergeordnete Formen eine vordergründige Rolle übernehmen, sodass randseitige Produktionen prototypische Merkmale generieren (55 f.). Dies gilt nicht nur für Psycho, dessen Motive eine Reihe von Horrorfilmen und Thriller geprägt haben und im historischen Genrediskurs prototypische Funktionen zugesprochen bekommen, sondern ist vor allem über die Sequels und Bates Motel zu beobachten, die sich explizit auf Psycho in seiner dominanten Genreposition berufen, aber zugleich eine generisch-serielle Umschichtung (Sequels) und Dekonstruktion (Bates Motel) dieser Merkmale vornehmen. Wie in der Analyse der intertextuellen Bezüge der Sequels und von Bates Motel deutlich wird, werden Motive und Szenen von Psycho zwar anzitiert, aber als ,historische Merkmale“ 
markiert und von neuen Genremerkmalen und Genresemantiken ,überschrieben“. Diese beinhalten unter anderem eine explizitere Gewaltdarstellung in den Sequels und eine Neuperspektivierung von Gendersemantiken in Bates Motel. Psycho's Merkmale werden dadurch aber nicht verdrängt. Insbesondere die Sequels schichten sie im Sinne Grindons zu subdominanten Merkmalen um und überführen sie in einen generisch-seriellen Intertext, in dem historische Merkmale von Psycho und gegenwärtige Genremuster koexistieren. Zwar leisten dies zuvor bereits Produktionen über intertextuelle Bezüge, aber die Sequels und die Prequel-Serie formieren einen seriellen Bezugsrahmen, indem diese generischen Veränderungen über wechselseitige Lektüren miteinander konfrontiert und sichtbar gemacht werden. ${ }^{19}$

Mit Grindons Ansatz lassen sich die Produktionen des Untersuchungskorpus über ihre Iterationen also sowohl in einer generischen Gesamtperspektive erfassen als auch in ihren spezifischen Ausprägungen als subgenerische Einheiten voneinander differenzieren. Dafür wird Psycho in seinen Genrediskursen untersucht, die einerseits die diskursive Komplexität von Genres auf der Ebene der einzelnen Genreproduktion in historischer Perspektive veranschaulichen und anderseits die Grundlage bilden für die anschließende Analyse der Sequels, die wiederum die historische Komplexität von Genres auf der Ebene einer Genregruppierung in diskursiver Perspektive beleuchten. Die Analyse von Bates Motel integriert Aspekte aus beiden Analysen, weil sich die diskursive und historische Komplexität von Genres durch die zeitliche Anordnung als zeitgenössische Prequel-Serie sowohl innerhalb des distributionsseitigen Genrediskurses als auch der generisch-seriellen Dekonstruktion der Duschszene zeigen lässt.

Die genretheoretischen Ausführungen zur diskursiven Beschaffenheit und zum historischen Wandel von Genres haben zentrale Aspekte verdeutlicht, die für die Analyse des Untersuchungskorpus von Relevanz sind. Inwiefern diese in einem analytischen Fokus auf Genresignaturen integriert werden können und welche spezifischen Fragestellungen sich für die analytischen Auseinandersetzungen ergeben, wird im Folgenden anhand einer knappen Zusammenfassung konkretisiert.

${ }^{19}$ Dieser serielle Bezugsrahmen wird hinsichtlich der Interdependenzen, die diese intertextuelle Umschichtung und generischen Veränderung bewirken und selbstreflexiv offenlegen, in Abschn. 4.1 theoretisch erarbeitet. 


\subsection{Aus dem Baukasten der Genretheorie und im Spiegel der Genregeschichte: Genresignaturen}

Die vorliegende Arbeit schlägt für die Analyse von Psycho, den Sequels und Bates Motel vor, sich aus dem Baukasten der Genretheorie sowohl eines historischen Genreverständnisses zu bedienen, das neben den wissenschaftlichen Konzeptualisierungen verstärkt die Diskurse der Filmkritik und Materialien in den Fokus rückt (Neale), als auch einen diskursiven Ansatz heranzuziehen, der für die Bezeichnungsebene sensibilisiert (Altman). Dass diese im Kontext kultureller Genrekonventionen entstehen (Tudor), wird hier ebenso einbezogen wie die kulturelle Normung von Prototypen, um die sich ein flexibles Genrebewusstsein spinnt (Schweinitz). Neben diesen übergeordneten Ansätzen gilt es, sich auf Iterationen in generischen Subeinheiten zu konzentrieren (Grindon), die in Bezug zu intertextuellen und phänomenologischen Einzeltheorien (Clover und Williams) generische Umschichtungs- und Dekonstruktionsvorgänge verdeutlichen. Für die Analyse der Diskurse und Iterationen wird zudem ein poststrukturalistisches Verständnis bedient, das von historischen Diskursformationen ausgeht, die ,systematisch die Gegenstände bilden, von denen sie sprechen“ (Foucault 74) und so den ,generischen Serien-/Text" wie auch , generisch-seriellen Intertext" als semantische Einheit erst erzeugen (Derrida).

Da jede Fallanalyse nicht nur auf einer anderen Genreebene operiert, sondern auch eine jeweils andere Methodik heranzieht, werden die jeweiligen Vorgehensweisen zu Beginn der Fallanalysen noch einmal in ihren analytischen Schwerpunkten eingeführt. Dies ermöglicht eine gezieltere Abstimmung auf das Material, indem im Vorhinein spezifische Analyseverfahren benannt werden: Für die diskursanalytische Betrachtung von Psycho und seiner Genrediskurse wird ein Überblick über die textbasierten Materialien zu Hitchcock und Psycho gegeben, um diskurshistorische Schwerpunkte zu identifizieren und für die anschließende Diskursanalyse produktiv zu machen. Für die Analyse der Iterationsmuster in den Sequels wird vorab ein intertextueller Genreansatz erarbeitet, in dem in Rückgriff auf Theorien zu Remakes und Serialität zentrale Differenzverfahren erarbeitet werden, die nicht nur die Iterationen zwischen den Sequels, sondern auch die Interdependenzen zu Psycho analytisch beschreibbar machen. Diese diskursanalytischen und intertextuellen Genreansätze werden in der Analyse von Bates Motel im Kontext serienspezifischer Dynamiken digitaler Serienkulturen fortgeführt, sodass keine neue Vorgehensweise erarbeitet wird.

Als diskursive Größe und historisches Phänomen bewegen sich Genres zwischen den ,generic images“ der Materialien (Neale), der Wirksamkeit eines kulturellen Genrebewusstseins und dem kulturkritischen wie wissenschaftlichen 
Bedürfnis nach generischer Genauigkeit auf der einen Seite und der Historizität von Genrebezeichnungen, semantischen Konzepten und Iterationsmustern auf der anderen Seite. Diese von Berry-Flint und Grindon beschriebene produktive Spannung kennzeichnet das wechselseitige Verhältnis von Genreproduktion und Genrekonzept und das Untersuchungskorpus der Arbeit. Mit dem Begriff der ,Genresignaturen's schlägt die vorliegende Arbeit eine analytische Perspektive vor, um die produktive Spannung von Genres sowohl in den einzelnen Fallanalysen als auch korpusübergreifend beschreibbar zu machen.

In der analytischen Begrifflichkeit der Genresignaturen verschränken sich die diskursive und historische Ebene der Untersuchungsmaterialien, indem sie auf der Ebene der Genreproduktionen die von generischen Markierungen und Iterationsmustern ausgehende Signifikanz beschreiben, die über die Diskursivierungen, ob diskursanalytisch im Kontext ihrer Entstehung betrachtet oder intertextuell in Bezug zu Genrekonzepten verortet, semantisch hergestellt wird. Zugleich geht der Signaturenbegriff auf die poststrukturalistische Theoriebildung von Derrida zurück. ${ }^{20}$ „Signaturen“ markieren einen Aufschub der Zeichen innerhalb eines allgemeinen Raums ihrer Möglichkeiten: „Dieser allgemeine Raum ist zunächst die Verräumlichung als Unterbrechung der Anwesenheit im Zeichen (marque) [...].“ (,Randgänge“ 348) Die Unterschrift verspricht eine Anwesenheit der Quelle und des Unterzeichnenden, die sie nicht halten kann. Die Anwesenheit ist für Derrida ebenso unmöglich, wie sie die Grundbedingung ihrer Existenzberechtigung darstellt:

Eine schriftliche Unterzeichnung impliziert per definitionem die gegenwärtige oder empirische Nicht-Anwesenheit des Unterzeichners. Aber, wird man sagen, genauso kennzeichnet und wahrt sie auch sein Anwesendgewesen-Sein in einem vergangenen Jetzt [...]. (Ebd. 349)

Eine Signatur bezeugt die absolute Einmaligkeit eines Ereignisses, das wiederum durch die Reproduzierbarkeit der Signatur zu Stande kommt. Um als Unterschrift

\footnotetext{
${ }^{20}$ Der Signaturbegriff ist Teil einer Auseinandersetzung mit dem Begriff der Kommunikation, die Derrida mit „Signatur Ereignis Kontext“ überschreibt (,Randgänge“ 325-351). Zur Bedeutung von Derridas Theorie für die Medientheorie siehe Fahle. Auch Deleuze bedient sich bereits der Signatur, allerdings um eine autorenbezogene Instanz im Film aufzubauen, die große Auteurs stilistisch in ihre Filme , einschreiben’ („Das Bewegungsbild-Bild“ 39). In diesem Deleuze'schen Sinn beschreibt Nitsche Hitchcocks Cameo-Auftritte als Signaturen (Nitsche 101-106). Dagegen verwendet Podrez den Signaturenbegriff in einem generischen Sinnzusammenhang, um die räumlichen Muster im Horrorfilm zu beschreiben (Podrez, „Der Horrorfilm“ 14 ff.).
} 
zu wirken, muss sie ,lesbar“ sein und ,eine wiederholbare, iterierbare, nachahmbare Form haben“ (ebd.). In dieser formalen Gleichheit widerspricht sie der Einmaligkeit des Produktionsereignisses, das sie herstellt und zugleich verfälscht. Für die Kommunikation heißt das nach Derrida, dass sich ,der semantische Horizont" nicht direkt ,überträgt" und nicht einfach als mehrdeutig herausstellt, also „,sich nicht auf eine Polysemie reduziert“ (ebd. 350). „Die Schrift liest sich“ (ebd.) und vermag darin keinen , eigentlichen Sinn" und keine ,tiefere Wahrheit" hervorzubringen. Damit erhält sich auch die Signatur aus der Trennung von der Quelle und in der Unmöglichkeit, einen semantischen Ursprung hervorzubringen. Zugleich schreibt sie sich in ihrer Gleichheit wiederholt fort, kann aber ihre Bedeutung nicht in einem Bezug zum Ursprung erlangen, sondern nur aus der Differenz zu diesem herstellen.

Auch der generische Text, liest sich" und offenbart darin keine generische Ursprungsbedeutung, wenngleich sich seine Markierungen und Iterationen wie Signaturen auf Genres als Bezugsquellen beziehen, die sie doch selbst erst hervorbringen. Genres sind Ursache und Effekt solcher ,Genresignaturen', die sich wiederholen und nur durch die Differenz zu dem zuvor Wiederholten erhalten. Wie oben an mehreren Stellen deutlich wird, führen Genres eine stete Differenz fort, die weder in einer genealogischen Identität zur Ruhe kommt, noch sich in eine geschlossene Einheit auflöst. Der generische Text drängt auf fortwährende diskursive und semantische Konstruktionsleistungen, die sich über Genresignaturen in ihrer Wiederholbarkeit und Differenzierung lesen lassen. Dieses Verständnis von Signaturen knüpft nicht nur an die im Zentrum der Untersuchung stehende diskursive und historische Verfasstheit von Genres an, sondern bietet auch einen übergreifenden analytischen Fokus, der die Mikroebene der Fallanalysen und die Makroperspektive der Genreuntersuchung wechselseitig perspektiviert und Differenzverfahren und Verschiebungsvorgänge verdeutlicht. Unter Genresignaturen werden dynamische Muster verstanden, die in signifikanter Weise die Genregeschichte von Psycho, den Sequels und von Bates Motel prägen und über eine diskursive und historische Varianz verfügen. Die Analysen zielen darauf, diese Varianz beschreibbar zu machen, indem sie Markierungen und Iterationen in den einzelnen Produktionen identifizieren und über die Kontextualisierung der Genrebezüge als semantische Einheiten und Differenzmomente diskursivieren. Das Korpus so entlang von Genresignaturen genrehistorisch zu erfassen, ermöglicht die Integration verschiedener Ansätze und analytischer Vorgehensweisen, um möglichst materialnah spezifische audiovisuelle Darstellungsformen und die umgebenden semantischen Differenzverfahren $\mathrm{zu}$ untersuchen. $\mathrm{Zu}$ betonen ist aber, dass das Konzept der Genresignaturen weniger als Einzelgenretheorie oder Metatheorie funktioniert, sondern vielmehr 
eine analytische Methodik bildet, die sich für ein eng gefasstes und exklusiv gebildetes Genrekorpus eignet, um exemplarisch die diskursive und historische Umschichtung von Genresemantiken entlang signifikanter Muster herauszuarbeiten. Für die Untersuchung von Psycho, den Sequels und Bates Motel lassen sich damit folgende Ausgangsfragen ableiten: Wie formieren Genrediskurse Psycho zu einem generischen Text und Bates Motel zu einem generischen Serientext? Wie bilden die Sequels und Bates Motel sich in ihren Wechselbezügen zu generischseriellen Intertexten heraus? Welche Bezeichnungen, Rhetoriken und Bezüge werden benutzt? Welche Markierungen, Iterationen und Semantiken lassen sich in den Materialien und Produktionen als Genresignaturen identifizieren?

In den Analysen kommt den distributionsseitigen Materialien in mehrfacher Hinsicht eine generische Schüsselposition zu: Sie führen die Herstellungs- und Publikumsebene, wenn auch indirekt und begrenzt, mit und gehen in einem multimedialen Verbund auf, in dem sie ihre jeweilige Medialität beibehalten. Zugleich bilden sie Genremerkmale aus, die als medienunspezifische Wanderphänomene eine reine Bezeichnungsfunktion übersteigen. Ihre audiovisuelle Gestaltung zielt auf eine produktspezifische Generizität, die die Produktion als einzelne ,Genreproduktion" von anderen abgrenzt. Doch diese Genrespezifik, das generic image von Neale, stellt sich erst über den Bezug zu kulturellen Konventionen her, die die einzelne Produktion wiederum mit anderen teilt. In den Materialien manifestiert sich damit die für Genres zentrale Differenz zwischen Spezifik und Konventionalität. Dieses Verhältnis zwischen einzelner Produktion und kulturellen Gemeinsamkeiten kennzeichnet die strukturelle Lücke von Genres. In der Unüberwindbarkeit, eine vollständige Übereinkunft zwischen Produktion und Konzept herzustellen, erzeugen Genres ein produktives Moment steter Reproduktion. An diesem Punkt zeigen sich Genrebezeichnungen im Bereich des Films auch in einem genealogischen Sinn als erste Vermittlungsinstanzen. Sie sind nicht nur erste diskursive Markierungen, die die Produktion mit einem Konzept (oder mehreren Konzepten) verbinden, sondern historisch einem Vertriebs- und Werbebedürfnis entwachsen und damit in ihrem ,Ursprung' auf Vermittlung angelegt. Genres verfügen über das adaptive Potential, Konventionen zu übernehmen, sich zu eigen $\mathrm{zu}$ machen und $\mathrm{zu}$ übermitteln. Angesichts ihrer genrespezifischen Relevanz werden die Materialien in den Fallanalysen zu Psycho und Bates Motel gleichwertig zu den Diskursen der Filmkritik und Film-/Genretheorie (bei Psycho) wie den iterativen Diskursivierungen (bei Bates Motel) behandelt, wo sie sich sogar als wirkungsmächtige Diskurse herausstellen. Einerseits verleihen sie den Genrekonzepten des Horror- wie Thriller-Genres durch doppelte Genremarkierungen und eine sensationsgeladene Aufbereitung eine, verquickende" neue 
Dynamik, für die Psycho paradigmatisch werden soll. Anderseits demonstrieren die Materialien von Bates Motel eine für digitale Serienkulturen zusätzliche Komplexitätssteigerung, die nicht nur das Verhältnis von Medien- und Werbeinhalten auf den Kopf stellt, sondern unter anderem auch Formen der kritischen Selbstbefragung generiert. Dagegen werden die Materialien in den Analysen zu den Sequels nicht herangezogen, weil der analytische Schwerpunkt auf den generisch-seriellen Iterationen liegt.

Das konstitutive Wechselverhältnis von Produktion und semantischem Konzept, das den strukturellen ,Motor ' von Genres bildet und sich in dem poststrukturalistischen Textverständnis niederschlägt, strukturiert auch die Analysen des Untersuchungskorpus maßgeblich. Psycho steht als einzelne Genreproduktion im Fokus, um den Genrediskurs in historischer Perspektive zu untersuchen und unter Einbezug semantischer Genrekonzepte diskursanalytisch aufzuarbeiten. Die Sequels formieren ein nach Grindon enges Korpus auf der Ebene des Genrekonzepts. Der für die Sequels herangezogene intertextuelle Ansatz setzt die Iterationen sowohl in Bezug zu Psycho als auch zu Clovers Theorie des Slasherfilms und Williams' Körpertheorie des Horrors. Schließlich gehen beide Ebenen und Ansätze in Bezug auf Bates Motel in einer integrativen Analyse zusammen, um sowohl den Genrediskurs entlang ausgewählter Materialien zur ersten Staffel zu beleuchten als auch die Iterationen mithilfe Clovers Theorie zu analysieren. Dieses Vorgehen deckt nicht nur die grundsätzliche Varianz und Dynamik von Genres auf, sondern verdeutlicht, wie die Produktionen in ihren Diskursen als ,generische Serien-/Texte' und in ihren Iterationen als ,generischserielle Intertexte" variabel formiert sind. So wird bei Psycho unter anderem Marions Perspektive durch die verregnete Windschutzscheibe als Markierung der Verunsicherung sowohl im Kontext des Thrillers als auch des Horrors identifiziert, mit der sich die Angst der Heldin auf die Zuschauenden überträgt und ein emotionaler Vorlauf für die kommende Schockwirkung des Duschmords geschaffen wird. Während in den Sequels der Duschmord als ,historische' Genresignatur nur anzitiert wird, um gegenüber den expliziten Mordszenen zum subdominanten Genremerkmal zu werden, , spielt‘ Bates Motel die Duschszene wieder vollständig aus, nicht ohne sie aber vorher in ihren Elementen zu dekonstruieren und erst anschließend unter einer neuen Gendersemantik zu einer dominanten, aber neuen queeren Genresignatur (wieder-)aufzubauen.

Insgesamt ermöglicht der Baukasten der Genretheorie die Zusammenstellung eines integrativen Theorie- und Methodendesigns, welches in den Analysen sowohl eine Makroperspektive auf die historischen wie diskursiven Veränderungen des Untersuchungskorpus aufrecht erhält als auch in den Mikroebenen der 
Fallanalysen die spezifischen Verfahren der Differenzierung herausarbeitet, wie unter anderem multiple Markierungen, wechselseitige Relektüren und genderbezogene Variationen. Inwiefern diese nicht nur die diskursive und historische Flexibilität von Genres exemplifizieren, sondern auf der Makroebene ein transgenerationelles Genregedächtnis ausbilden, wird in den folgenden Fallanalysen mit Blick auf die Genresignaturen untersucht.

Open Access Dieses Kapitel wird unter der Creative Commons Namensnennung 4.0 International Lizenz (http://creativecommons.org/licenses/by/4.0/deed.de) veröffentlicht, welche die Nutzung, Vervielfältigung, Bearbeitung, Verbreitung und Wiedergabe in jeglichem Medium und Format erlaubt, sofern Sie den/die ursprünglichen Autor(en) und die Quelle ordnungsgemäß nennen, einen Link zur Creative Commons Lizenz beifügen und angeben, ob Änderungen vorgenommen wurden.

Die in diesem Kapitel enthaltenen Bilder und sonstiges Drittmaterial unterliegen ebenfalls der genannten Creative Commons Lizenz, sofern sich aus der Abbildungslegende nichts anderes ergibt. Sofern das betreffende Material nicht unter der genannten Creative Commons Lizenz steht und die betreffende Handlung nicht nach gesetzlichen Vorschriften erlaubt ist, ist für die oben aufgeführten Weiterverwendungen des Materials die Einwilligung des jeweiligen Rechteinhabers einzuholen.

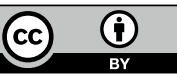

